\title{
Cis acting variation is common, can propagates across multiple regulatory layers, but is often buffered in developmental programs
}

$$
\begin{gathered}
\text { Swann Floc'hlay, }{ }^{1, *} \text { Emily Wong } \\
\text { 2,3* } \\
\text { Rebecca R. Bingqing Zhales }{ }^{4} \text {, Morgane Thomas-Chollier }{ }^{1,5}, \text { Denis Thieffry }^{1} \text {, } \\
\text { David A. Garfield }{ }^{4, \dagger} \text {, and Eileen EM Furlong }{ }^{4, \dagger}
\end{gathered}
$$

${ }^{1}$ Institut de Biologie de l'ENS (IBENS), École Normale Supérieure, CNRS, INSERM, Université PSL, 75005 Paris, France.

${ }^{2}$ Molecular, Structural and Computational Biology Division, Victor Chang Cardiac Research Institute, Darlinghurst, New South Wales, Australia

${ }^{3}$ School of Biotechnology and Biomolecular Sciences, UNSW Sydney, Kensington, New South Wales, Australia

${ }^{4}$ European Molecular Biology Laboratory (EMBL), Genome Biology Unit, D-69117, Heidelberg, Germany

${ }^{5}$ Institut Universitaire de France (IUF), 75005 Paris, France.

*These authors contributed equally, listed alphabetically

†To whom correspondence should be addressed E-mails@dagarfield@gmail.com, furlong@embl.de Telephone +4962213878416 Fax $\quad+4962213878166$

Running title: Dissecting the functional impact of DNA variation

Keywords: Natural sequence variation, developmental enhancers, F1 embryos, open chromatin, transcription, gene regulation, selection 


\section{Abstract}

10 Precise patterns of gene expression are driven by interactions between transcription factors,

11 regulatory DNA sequence, and chromatin. How DNA mutations affecting any one of these

12 regulatory 'layers' is buffered or propagated to gene expression remains unclear. To address

13 this, we quantified allele-specific changes in chromatin accessibility, histone modifications, and

14 gene expression in F1 embryos generated from eight Drosophila crosses, at three embryonic

15 stages, yielding a comprehensive dataset of 240 samples spanning multiple regulatory layers.

16 Genetic variation in cis-regulatory elements is common, highly heritable, and surprisingly

17 consistent in its effects across embryonic stages. Much of this variation does not propagate to

18 gene expression. When it does, it acts through $\mathrm{H} 3 \mathrm{~K} 4 \mathrm{me} 3$ or alternatively through chromatin

19 accessibility and H3K27ac. The magnitude and evolutionary impact of mutations is influenced

20 by a genes' regulatory complexity (i.e. enhancer number), with transcription factors being most

21 robust to cis-acting, and most influenced by trans-acting, variation. Overall, the impact of

22 genetic variation on regulatory phenotypes appears context-dependent even within the constraints

23 of embryogenesis. 


\section{Introduction}

25 The development of a multicellular organism requires the tight regulation of gene expression

26 in both space and time to ensure that reproducible phenotypes are obtained across individuals and environmental conditions. DNA regulatory elements (e.g. promoters and enhancers) are essential to this process by integrating regulatory information from sequence-specific transcription factors (TFs), RNA polymerase II (Pol II), and other regulatory proteins to drive specific spatio-temporal patterns of expression during development. But while gene expression patterns are typically quite precise, the DNA regulatory elements that control such expression states are replete with genetic variation (mutations) that can impact transcriptional regulation at multiple levels including TF binding (Kasowski et al. 2010; Spivakov et al. 2012; Behera et al. 2018), chromatin state (Waszak et al. 2015), transcriptional start site usage (Schor et al. 2017), gene expression levels (Garfield et al. 2013; Battle et al. 2015), and transcript isoform diversity (Cannavo et al. 2017). As a result, genetic variation in regulatory elements can contribute to variation in disease susceptibility among individuals (Epstein 2009; Lowe and Reddy 2015) and to evolutionary change between species (Wittkopp and Kalay 2011), by impacting higher-level phenotypes.

Although regulatory mutations can have large effects, many behave effectively neutrally, making it challenging to predict which genetic variants will have an impact. Part of the difficulty comes from the general lack of knowledge about which regions of non-coding DNA have regulatory (not just biochemical) function. An additional challenge is the apparent robustness of gene regulatory networks. At least within a laboratory context, whole sections of regulatory DNA can be removed with little apparent impact on phenotype or fitness (Ahituv et al. 2007), and evolutionarily divergent regulatory sequences are often swapped between species with few detectable changes in gene expression (Borok et al. 2010). These studies demonstrate that developmental systems have the ability to compensate or "buffer" the effects of regulatory mutations, e.g. via compensation by other regulatory elements with partially overlapping activities (Hong et al. 2008; Frankel et al. 2010; Cannavo et al. 2016).

The complex relationship between DNA sequences and regulatory function further complicates our understanding of how mutations can impact gene regulation. For example,

52 mutations affecting TF binding motifs can have a large impact on chromatin accessibility, Pol II

53 occupancy, histone modifications and gene expression (Kircher et al. 2019). But in some 
54 contexts/tissues, TF binding is driven by collective processes that can include protein-protein as 55 well as protein-DNA interactions, such that mutations affecting a single TF motif may not 56 substantially affect TF recruitment (Junion et al. 2012; Doitsidou et al. 2013; Uhl et al. 2016;

57 Khoueiry et al. 2017). Moreover, many sequence variants affecting TF occupancy in vivo lie 58 outside the TF's binding motif, and are likely due to variation affecting the binding of cooccurring factors (Kasowski et al. 2010; Zheng et al. 2010; Reddy et al. 2012) or an overall

60 change in DNA shape (Lu and Rogan 2018). To make matters more complex, enhancer output is 61 not a strict function of all TF's occupancy - enhancers often contain binding sites for multiple 62 factors with redundant input, and in some cases, different combinations of TFs can produce the 63 same expression output (Brown et al. 2007; Zinzen et al. 2009; Khoueiry et al. 2017). Even in 64 cases where an enhancer's activity is abolished by mutations, the gene's expression may not be 65 affected, as genes often have many enhancers with partially overlapping activity, which can 66 buffer the functional impact of genetic variation impacting a single enhancer (Hong et al. 2008;

67 Frankel et al. 2010; Cannavo et al. 2016). With a few exceptions (Bullaughey 2011), this 68 complex genotype-to-phenotype relationship cannot be modelled using regulatory sequence 69 information alone, but rather must be evaluated empirically (Khoueiry et al. 2017).

Allelic-specific data provides a unique opportunity to study the molecular mechanisms of cisacting variation and has uncovered multiple regulatory processes through which cis-acting variation impacts transcriptional control (Kilpinen et al. 2013; Chen et al. 2016). F1 crosses of inbred strains provide an elegant method to determine the contribution of both cis and trans variation by overcoming the limits of genetic variation between trios and the general lack of statistical power to interrogate trans-acting variation using population data (Wittkopp et al. 2004; Tirosh et al. 2009; Goncalves et al. 2012; Wong et al. 2017). Taking advantage of this F1 design, we set out to better understand how natural sequence variation impacts gene regulation during embryonic development. We collected Drosophila F1 hybrid embryos and quantified allelespecific changes in open chromatin (ATAC-Seq), enhancer and promoter activity (using

$80 \mathrm{H} 3 \mathrm{~K} 27 \mathrm{ac}$ or H3K4me3 \& H3K27ac ChIP-Seq as proxies, respectively), and gene expression 81 (RNA-seq). Our half-sibling design of F1 embryos was generated by crossing males from eight 82 genetically distinct, wild-derived isogenic lines from the Drosophila Genetic Reference Panel 83 (DGRP) (Mackay et al. 2012) to females from a common, laboratory-derived isogenic reference 84 strain. In addition to having practical advantages for conducting large scale crosses, as described 
85 below, the use of a common female line allowed us to evaluate the impact of regulatory

86 mutations while controlling for maternal effects, which can contribute disproportionately to

87 variability in early developmental phenotypes (Lynch and Walsh 1998; Garfield et al. 2013). By

88 collecting matched phenotypic measurements from two parental strains $(F 0)$, we also estimated

89 the heritability of cis-acting mutations and the relative magnitude of trans-acting genetic

90 variation that contributes to phenotypic divergence.

91 Overall, we find allelic variation in chromatin accessibility and histone marks to be common

92 and significantly correlated between regulatory layers, with the effects of regulatory mutations

93 being more strongly coupled at promoters than distal elements (putative enhancers). Using this

94 genetic variation as a perturbation to gene regulation, we uncovered different mechanistic rules

95 in the order of information flow during transcriptional regulation. Specific classes of genes, such

96 as TFs, are in general more strongly buffered against the effects of this variation, which in turn

97 reflects their patterns of inheritance and genetic architecture (having a greater proportion of trans

98 and less additive heritability). In some cases, selection is driven to near fixation in gene

99 expression (but interestingly not in upstream regulatory layers), affecting genes involved in

100 environmental responses and pesticide resistance. Taken together, this comprehensive data set

101 provides new insights into the functional impact of cis-regulatory DNA variation and how this is

102 transmitted across different regulatory layers during embryogenesis, and how patterns of

103 inheritance can influence the visibility of regulatory sequence variants to natural selection. 


\section{Results}

\section{Quantifying gene expression and regulatory element activity in hybrid embryos}

107 We collected F1 hybrid embryos by mating eight genetically distinct inbred lines from the 108 DGRP collection (Mackay et al. 2012) to females from a common maternal line (Fig. 1a). The

109 resulting F1 panel contains an average of 567,412 SNPs per cross, and a total of 1,455,988

110 unique SNPs covering a range of minor allele-frequencies and levels of conservation (phyloP 111 scores) (Fig. S1a, Table S1).

112 The F1 embryos were collected at three important stages of embryogenesis: 2-4 hours after 113 egg laying, consisting primarily of pre-gastrulation, unspecified embryos (mainly stage 5), 6-8

114 hours (mainly stage 11), when major lineages within the three germ-layers are specified, and 10-

11512 hours (mainly stage 13), during terminal differentiation of tissue lineages (Fig. 1a). For each

116 developmental stage, RNA-Seq, ATAC-Seq, and iChIP for H3K27ac and H3K4me3 (Buenrostro

117 et al. 2013; Lara-Astiaso et al. 2014) were performed from the same collection of embryos (4

118 measurements $\times 3$ stages $\times 8$ genotypes $=96$ samples). In addition, we collected samples from

119 the parents of one F1 genotype, forming a parent/offspring trio that allowed us to partition

120 genetic differences between the parents into cis and trans (Wittkopp et al. 2004). All

121 measurements were made in replicates from independent embryo collections to assess biological

122 and technical variability, giving a total of 240 samples (192 F1 samples (96 x 2 replicates) +48

123 parental (4 measurement x 3 stages x 2 genotypes x 2 replicates)). Read counts were highly

124 correlated between biological replicates, with median correlation coefficients of 0.98 for RNA,

125 ATAC and histone data (Fig. S1b, Methods).

126 To define non-coding features, ATAC-Seq and ChIP-Seq reads from each cross were mapped 127 to each parental line independently and the significant peaks merged to produce a combined set 128 of common peaks used in subsequent comparisons across all genotypes. In total, we identified

129 11,211 genes with detectable expression, 31,963 ATAC-Seq peaks, 19,769 H3K27ac peaks, and $1306,648 \mathrm{H} 3 \mathrm{~K} 4 \mathrm{me} 3$ peaks, active at one or more stages of embryogenesis (Table S2). Of these, $13193.9 \%, 95.8 \%, 95.2 \%$, and 96.9\%, respectively, contained at least one SNP that distinguishes 132 maternal and paternal haplotypes in at least one line. The CG12402 locus, a predicted ubiquitin133 protein transferase, provides a good example of overall signal quality (Fig 1a). The gene has

134 dynamic expression, transitioning from very low to high expression from 2-4 hours to 10-12 
135 hours (Fig. 1a, RNA-seq), which is accompanied by quantitative changes in chromatin

136 accessibility, and to a lesser extent in histone modifications in its promoter-proximal region.

A

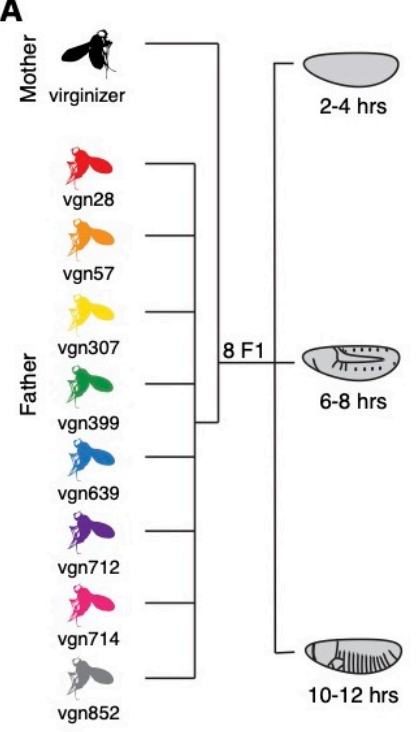

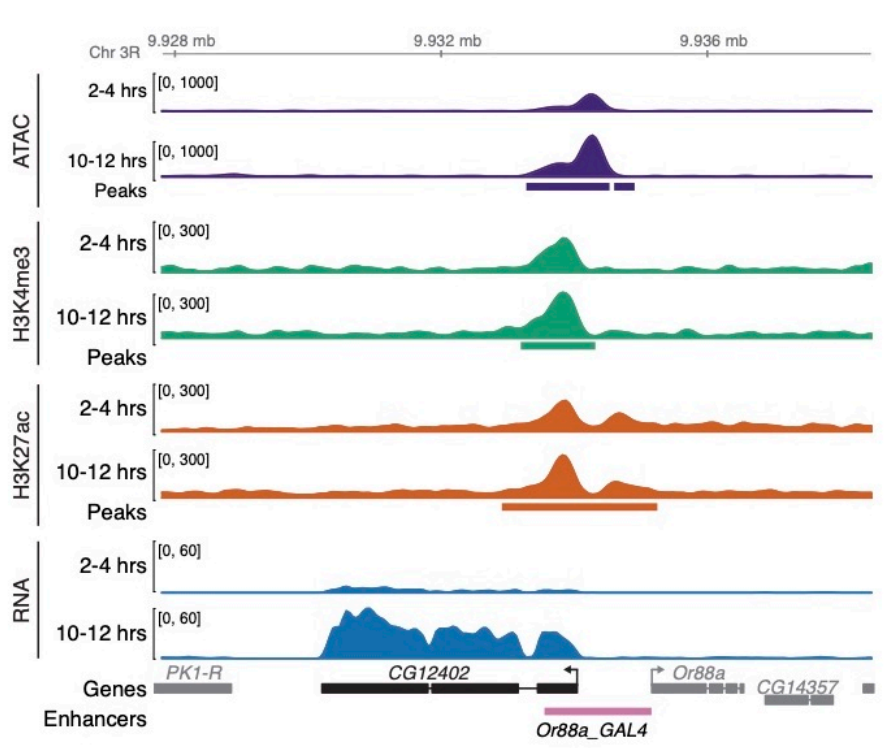

B
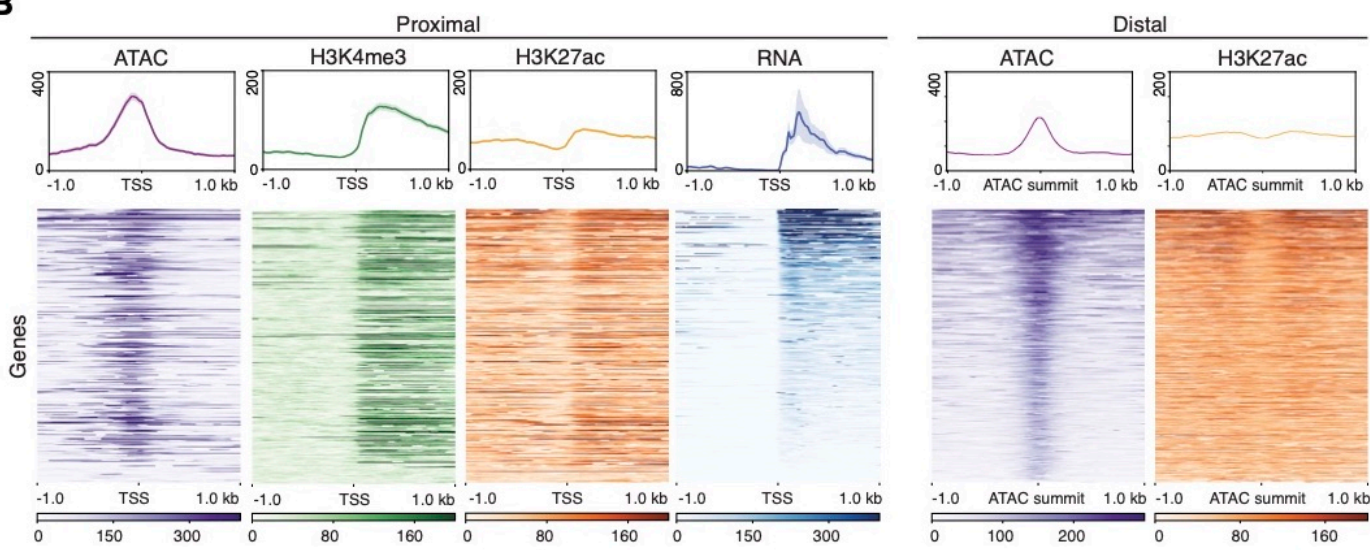

C
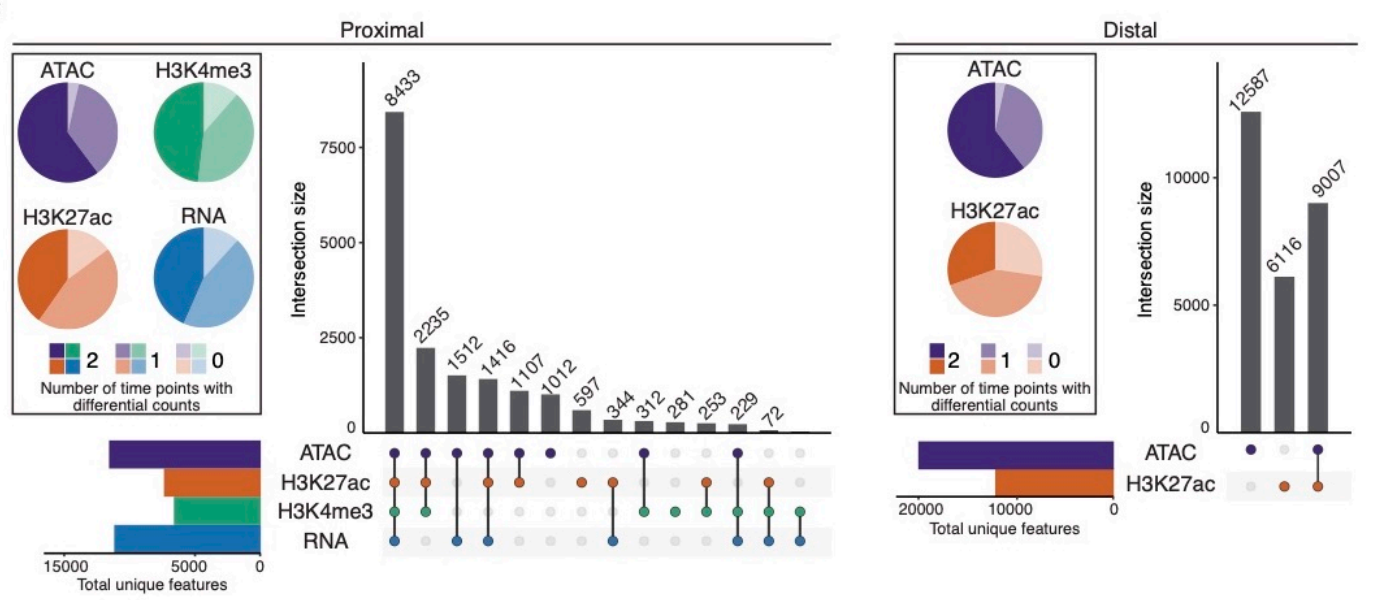


\section{Figure 1: Quantifying gene expression and regulatory element activity in hybrid embryos}

a. Left: Experimental design and data structure. RNA-seq, ATAC-seq and iChIP of H3K4me and H3K27ac were performed on embryos of three developmental stages from $8 \mathrm{~F} 1$ hybrids with a common maternal line. Right: Genome browser overview for the CG12402 gene locus showing all data for 2-4 hours and 10-12 hours for the genotype vgn28. Bottom track shows characterized enhancers (Kvon et al. 2014). b. Top panel shows density plots for read count signal from each data type for TSS proximal and distal regions (left and right, respectively). Shaded regions indicate the $95 \%$ confidence intervals. Plots are centered at the TSS for promoter proximal regions, and ATAC summits for distal regions. Bottom panel shows a heatmap representation of the data type corresponding to the density plots shown above where rows are sorted by mean RNA-seq and mean ATAC-seq signal. c. Upset plots show the colocalization of signal for proximal and distal regions (at peaks in regulatory regions and genes) for all four data types. Regions common between data types (filled circle) are joined by a vertical bar. Horizontal bar plots indicate the number of unique genes/features. Pie charts show the proportion of features with statistically different total read counts between time points (color indicates the number of times $(0 / 1 / 2)$ the feature is differentially expressed).

To examine the regulatory relationships between these different signals, we divided the data into promoter proximal (within $+/-500 \mathrm{bp}$ of an annotated transcriptional start site (TSS) or H3K4me3 peak) or distal (putative enhancer) elements. Looking globally at promoter proximal regions, all signals show the expected enrichment and distribution around the TSS (Fig 1b, proximal), demonstrating the quality of the data. The ATAC-seq signal, for example, is highest directly at the promoter, representing occupancy of the basal transcriptional machinery, while $\mathrm{H} 3 \mathrm{~K} 27 \mathrm{ac}$ and $\mathrm{H} 3 \mathrm{~K} 4 \mathrm{me} 4$ signals are highest at the +1 nucleosome, reflecting the predominantly unidirectional nature of Drosophila promoters (Core et al. 2012; Mikhaylichenko et al. 2018). Moreover, H3K27ac has the expected higher signal levels at promoters compared to distal sites, (Kheradpour et al. 2013; Kwasnieski et al. 2014). Interestingly, while all three regulatory signals (ATAC-seq, H3K27ac and $\mathrm{H} 3 \mathrm{~K} 4 \mathrm{me} 3$ ) are highly correlated at the promoters of actively transcribed genes (8,433 promoters contain all 4 signals, Fig 1c, left upset plot), 3,907 regions marked by $\mathrm{H} 3 \mathrm{~K} 4 \mathrm{me} 3$ and overlapping peaks of ATAC-seq and/or H3K27ac show no detectable RNA-signal (Fig. 1c bar plots, 1b). Approximately 850 of these involve annotated transcripts of non-coding RNA (Flybase annotation) that lack a poly-A tail and were thus not selected in our

152 Poly-A+ RNA-seq library. This suggests a surprising number of additional unannotated

153 transcriptional events even within the well-annotated Drosophila genome.

154 The majority of H3K27ac (62.5\%) and ATAC peaks (63.7\%) are distal to an annotated 155 promoter, and likely represent enhancer elements. Of the distal ATAC peaks, 58\%

$156(12,587 / 21,594)$ have no H3K27ac signal and may represent inactive enhancers or other 157 regulatory elements, e.g. insulators (Fig. 1c). The remaining 9,007 distal elements overlap $158 \mathrm{H} 3 \mathrm{~K} 27 \mathrm{ac}$ signal (Fig. 1c, right), which is generally bimodally distributed around the ATAC-seq 
peak (Fig. 1b), suggestive of active enhancers. Conversely, 40\% $(6,116 / 15,123)$ of H3K27ac

peaks do not overlap a significant ATAC peak (Fig. 1c, right) and represent regions with quantitively lower ATAC signal (Fig. S1c), below our stringent threshold for detection. Both gene expression (RNA-seq) and non-coding elements (based on ATAC-seq and chromatin signatures) show evidence of dynamic activity, with the majority (72\%-96\%) of features showing statistically significant changes in total counts between developmental time points across all $F 1$ lines (Fig. 1c, pie charts; Methods), CG12402 being one example (Fig. 1a). usefulness to further annotate the regulatory landscape of the Drosophila genome at these important stages of embryogenesis.

\section{Allele-specific variation is common across genotypes and regulatory layers}

171 To examine the impact of genetic variation, reads from each cross were mapped to personalized genomes for each parent and assigned to the maternal or paternal haplotype, where possible (Methods). To test for allele-specific differences for each gene per line and time combination, we used an empirical Bayes framework to model allele-specific counts for each data type using a beta-binomial model (Fig. S2a). Most promoter proximal and distal elements had the expected allelic ratios centered at 50:50 across autosomes (Fig 2a), with a slight elevation in the magnitude of allelic imbalance (AI) at distal sites (Fig. S2b). RNA allelic ratios were also concordant with the direction of change of embryonic eQTL (Fig. S2c), previously quantified in the same paternal lines at the same stages of embryogenesis (Cannavo et al. 2017), further verifying our approach.

181 To evaluate sex ratios in the embryo pools, and to set a reference point for evaluating allelic 182 imbalance and dosage compensation on the X-chromosome (Lucchesi and Kuroda 2015), we 183 sequenced the genomic DNA (gDNA) of each cross. This confirmed that our embryonic pools 184 were relatively sex balanced, with the expected X-chromosome allelic ratio of $\sim 0.66$ observed 185 across our gDNA dataset (Fig 2b). Consistent with full dosage compensation on the maternally-

186 derived male X chromosome (Georgiev et al. 2011), we observed a maternal:paternal ratio of

1870.74 for RNA (Fig. 2b; Methods). Interestingly, a similar degree of up-regulation (dosage

188 compensation) was not observed for chromatin data: for both chromatin accessibility and 
A

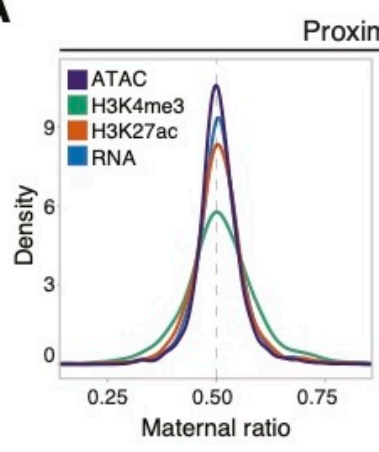

Proximal

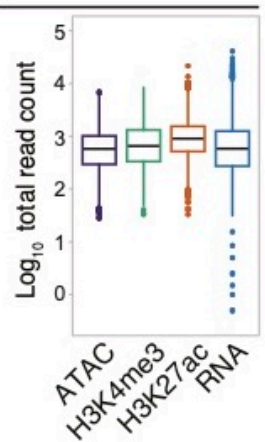

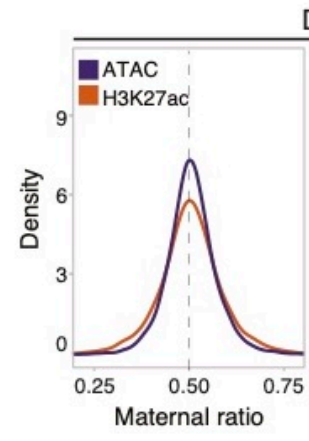

Distal

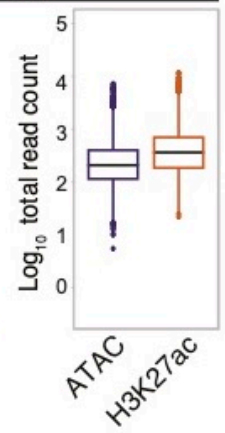

B

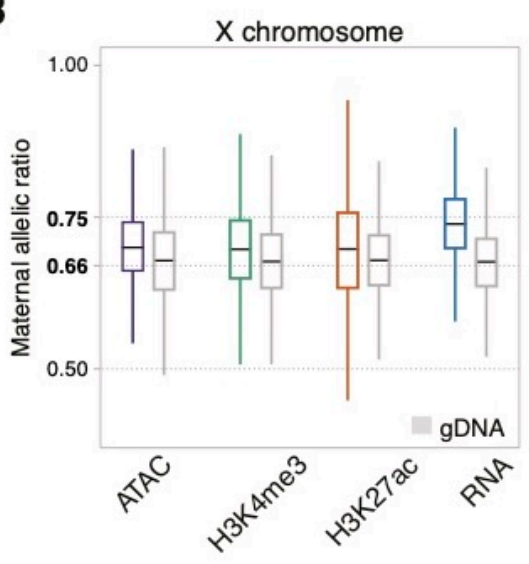

D

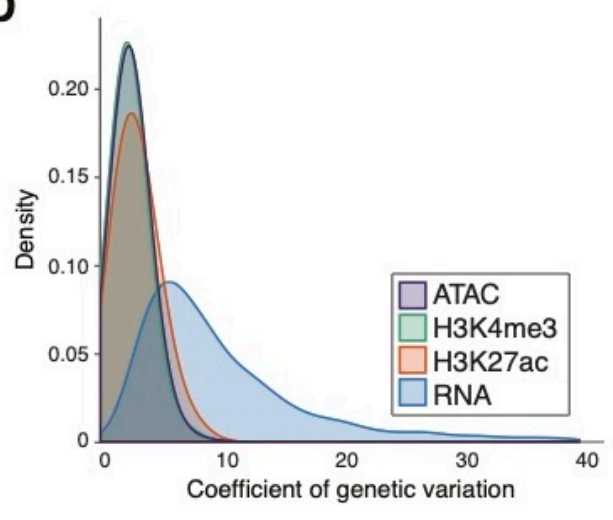

C

$$
\text { Allelic imbalance: }
$$
in at least one line at any time point

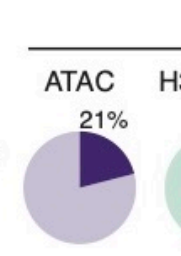

Proximal

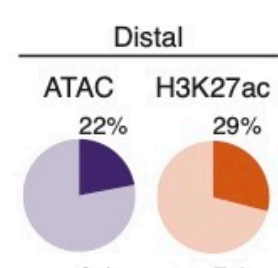

Allelic imbalance: each feature per line and time
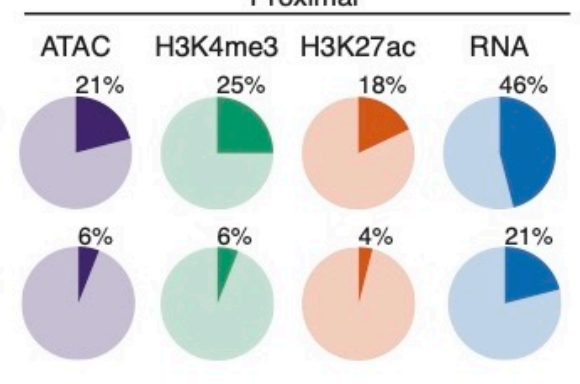

E
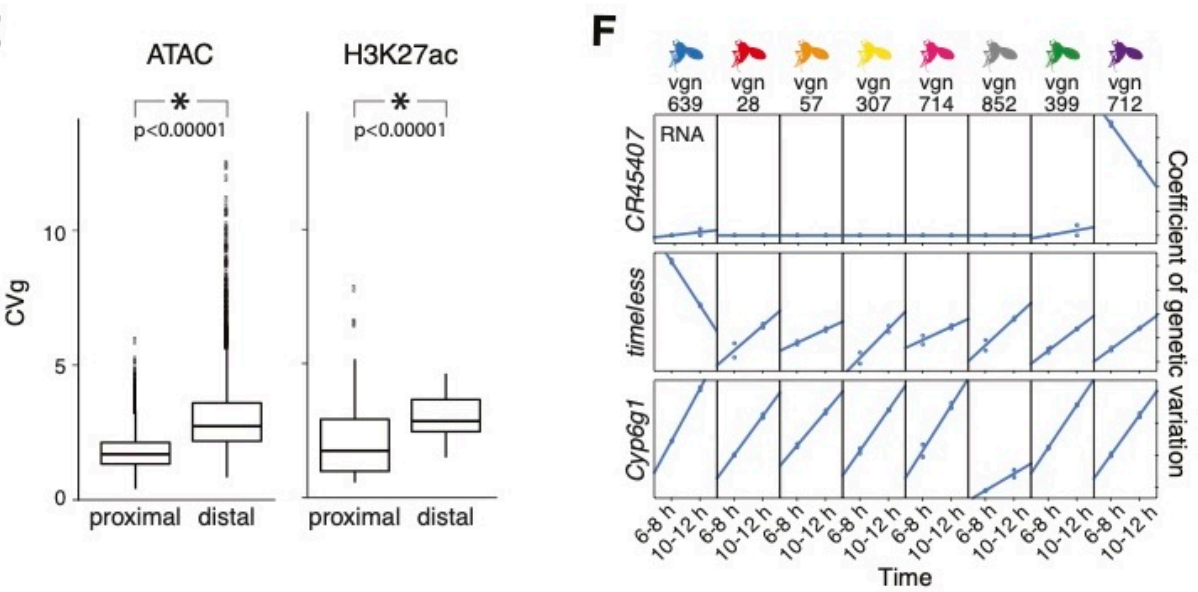


\section{Figure 2: Allelic imbalance is common across regulatory data types}

a. Density plot of allelic count distribution and matching boxplot showing total read count abundance $\left(\log _{10}\right)$ in the autosomes at TSS proximal (left) and distal (right) regions for all data types assayed. b. Box plot shows the distribution of the maternal allelic ratio of X chromosome in each data type. Each distribution is compared to the allelic ratio observed in genomic DNA in grey. c. Pie charts show significantly allelic imbalance (AI) genes/features at promoter proximal (left, TSS +/-500 bp) and distal (right, 500-1500 bp +/from TSS) regions for all four data types (FDR $<0.1)$. Upper: AI events in at least one F1 line at any time point. Lower: AI events detected in all $8 \mathrm{~F} 1$ lines in all time points, on a per line and time basis. d. Smoothed histograms show the distribution of coefficients of genetic variation for all features with statistically coefficient of genetic variation (CVg, y axis) for chromatin accessibility (left) and H3K27ac signal (right), for promoter -proximal and -distal sites. Genetic influences are more pronounced at distal regulatory elements in ATAC and H3K27ac. f. Line plots show three examples of individual lines having distinct expression profiles. Coefficients of genetic variation are typically larger for RNA than for non-coding features, an effect that often results from one or two lines having significantly altered expression relative to the panel as a whole.

histone modifications, the observed ratio at $\mathrm{X}$ chromosome sites $(\mathrm{H} 3 \mathrm{~K} 27 \mathrm{ac}=0.688$,

$\mathrm{H} 3 \mathrm{~K} 4 \mathrm{me} 3=0.692$ ) is more similar to the observed genomic ratio of 0.66 than to the expected ratio of 0.75 under full dosage compensation (Fig. 2b). The ratios showed no significant difference when comparing proximal to distal sites, arguing against the hypothesis that the twofold upregulation of gene expression on the male Drosophila X chromosome results from a two-

212 fold increase in the loading of polymerase at its genes' promoters (Conrad et al. 2012). Our

213 results rather indicate that whatever the mechanism of dosage compensation in Drosophila, it

214 does not lead to a linear increase in chromatin accessibility on the male X chromosome, though

215 some increase in accessibility on the upregulated $\mathrm{X}$ is consistent with our measurements (Urban

216 et al. 2017; Pal et al. 2019). Regardless of its cause, we used the empirically observed average

217 ratio for X-chromosome features for each data type to form the null-hypothesis in subsequent

218 beta-binomial tests for allelic imbalance.

219 Overall, allelic imbalance is common, with $46 \%$ of genes and between $18-25 \%$ of non-coding 220 features showing statistically significant AI in at least one line at one or more time point (Fig. 2c, 221 FDR $<0.1$ ). The magnitude of AI is generally evenly distributed across SNPs with a range of 222 minor allelic frequencies, however highly imbalanced peaks show a strong enrichment for 223 extremely rare SNPs (including potentially de-novo mutations) found uniquely in the maternal

224 line relative to the 205 lines of the full DGRP panel (Fig. S2d, Chi^ 2 test; $\mathrm{p}<2.2 \mathrm{e}-16$ ),

225 highlighting the disproportionate impact of rare and de-novo mutations on expression phenotypes 
226 (Cannavo et al. 2017). Allelic imbalance is more frequently observed for RNA than for other

227 regulatory layers (Fig. 2c). In contrast to what is observed in mammals (Villar et al. 2015),

228 promoter-proximal elements are slightly more polymorphic (pair-wise differences (pi) $=0.132 \mathrm{vs}$

2290.129 , Wilcoxon-test $\mathrm{p}=1 \mathrm{e}-10$ ) and evolve faster (phyloP $=0.514$ vs 0.560 , Wilcoxon-test, $\mathrm{p}<$

230 2.2e-16) in Drosophila as compared to distal elements (putative enhancers) (Table S3). Despite

231 this, distal peaks of open chromatin and H3K27ac show larger (Tukey's ASD, p < 0.0001)

232 and more frequent $\left(\chi^{2}\right.$ test, $\left.\mathrm{p}<2.2 \mathrm{e}-16\right)$ allelic imbalance than their proximal counterparts (Fig

233 S2b).

234 To understand how allelic-imbalance relates to heritable variation at the total count level, we 235 took advantage of the fact that our measured F1 lines share a common maternal genotype. As a 236 result, line effects (from a linear model) are expected to be directly proportional to heritability -

237 the degree to which phenotypic variation can be explained by genetic factors (Lynch and Walsh 238 1998). To make these effects comparable across genes and features, line effects (standard 239 deviations) were scaled by the mean read count of each feature, expressing line effects as a 240 percentage deviation from the mean phenotype (coefficient of genetic variation). For chromatin 241 features, the magnitude of genetic variation on measured signal is relatively modest, with the 242 average peak varying by $\sim 5-10 \%$ of the mean phenotype among crosses (Fig. 2d). Overall, the

243 effects of heritability genetic variation is higher at distal regulatory elements as compared to their

244 proximal counterparts (Fig. 2e, p < 1e-5, Methods), consistent with the greater magnitude of AI 245 at distal sites. For RNA, in contrast, the magnitude of genetic effects is pronounced, with an 246 average coefficient of genetic variation of $\sim 9 \%$ and some genes showing coefficients of $\sim 40 \%$,

247 indicating that genetically encoded differences in expression can account for nearly half of some 248 genes' mean expression levels. In many cases, such high coefficients are driven by one or a few 249 lines showing highly divergent patterns of expression (Fig. 2f, right) suggesting that large-effect 250 gene expression differences in this population can be driven by large-effect cis-acting mutations.

\section{Allelic imbalance is pronounced in metabolism and environmental response genes}

252 Imbalanced genes and associated regulatory features are enriched for fast-evolving and 253 Drosophila-specific genes lacking clear categorical annotations (Mi et al. 2003; Turner et al. 254 2008) and are depleted in TFs and their associated regulatory elements (Fig. S3, Table S4), 
consistent with our previous eQTL study (Cannavo et al. 2017). AI is also enriched for

metabolic genes at the RNA level, although interestingly this is not observed for associated

differences in AI among gene categories may reflect differential histories of selection; regulatory regions in the vicinity of TFs show a depletion of nucleotide diversity (pi, rank biserial correlation $=-0.052, \mathrm{p}<1 \mathrm{e}-4)$ and harbor more low-frequency SNPs (rank biserial correlation $=$ $-0.173, p=2.8 \mathrm{e}-3$; Table S4) compared to background. However, this difference AI could also be explained by different gene categories having different sensitivities to mutations (buffering), a point we explore further below.

264 For most gene categories, AI is equally likely to favor the maternal or the paternal allele. However, a subset of categories shows consistent and often large parent-specific biases, a trend that is particularly striking for male-biased genes associated with immunity or insecticide resistance (Fig. S4a; Table S5). Cyp6g1, for example, is not expressed in embryos of our maternal line (Fig. S4b), which is derived from a laboratory stock isolated before the widespread use of agricultural pesticides, or in embryos sequenced by the modENCODE project (Celniker et al. 2009). It is, however, strongly upregulated in every measured paternal haplotype from the wild, and its overexpression contributes to DDT resistance in multiple Drosophila species (Fig. S4b, (Daborn et al. 2001; Battlay et al. 2016)). Highly imbalanced genes like Cyp6g1 (Table S5) overlap genes whose expression varies extensively among lines (Fig. S4c, p < 1e-6) and who have high levels of heritability, highlighting the important contribution of selection on cisregulatory elements in shaping responses to changing environments.

\section{The impact of cis-acting genetic variation is largely consistent across development}

278 We next evaluated if, and to what extent, allelic ratios change during development. Overall, we observed a surprising constancy of allelic imbalance between embryonic time points: Despite

280 the temporal modularity of many cis-regulatory elements, imbalanced features at one time point

281 have a $\sim 50 \%$ chance of being imbalanced in the subsequent time-point (Fig. 3a, S5a). To further

282 quantify the potential impact of development on allelic ratios, we constructed a series of linear

283 models comparing the effect sizes of genetics (genotype/line effect) vs developmental stages

284 (time effect) on total counts and allelic ratios across our experimental design. For total counts, 
285 developmental time was the greatest contributor to variation across all data types (Fig. 3b, upper panel), consistent with the clear time specific clustering by principal component analysis (Fig. $3 \mathrm{~b}$, lower panel, shown for RNA, Methods). Interestingly, this predominance of time is largely restricted to distal and not proximal sites for ATAC-Seq (Fig 3d), likely reflecting the frequently constitutive accessibility of promoters during Drosophila embryogenesis (Cusanovich et al. 2018). In contrast to the total counts, the impact of developmental time on allelic ratios is

291 significantly reduced compared to genetic (line) effects (Fig 3c, upper panel). Correspondingly, there is a lack of time-point specific clustering in PCA (Fig 3c, lower panel), although there are some examples of allelic ratios that change over time in a coordinated manner between

294 regulatory layers (Fig. S5b).
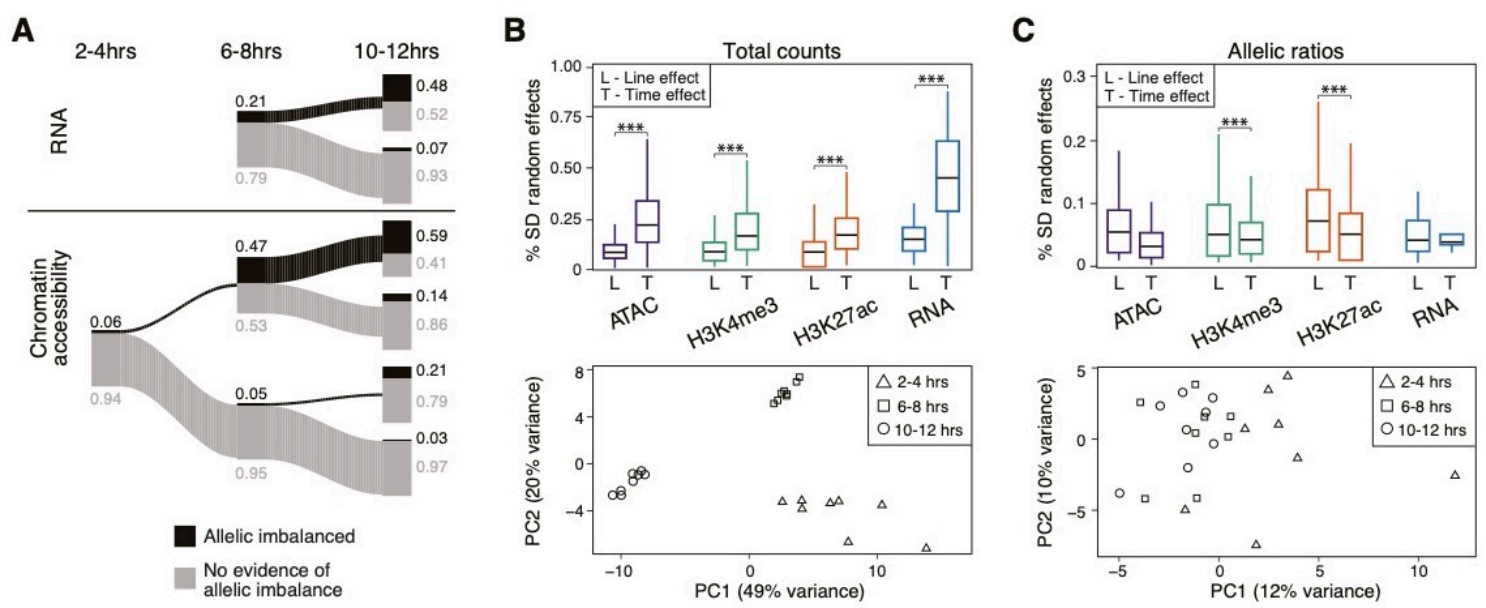

D
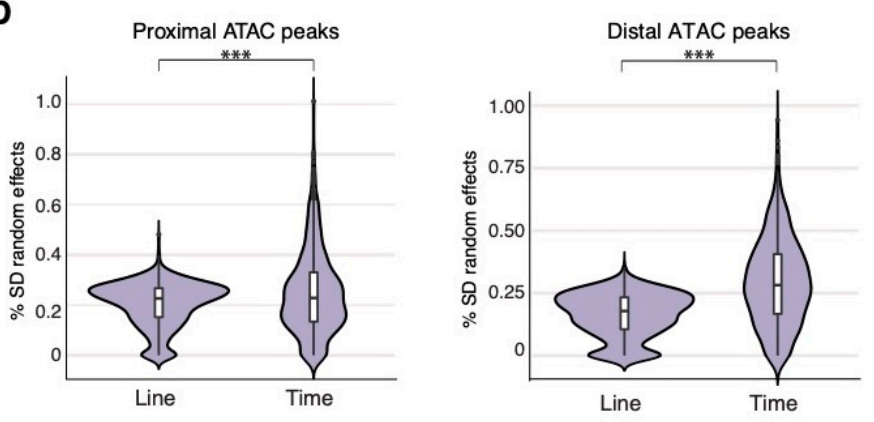

Figure 3: Allelic imbalance is generally not predictive of developmental time

a. The relationship of allelic imbalance across time points for RNA (upper panel) and chromatin accessibility (lower panel). Proportions of AI and non-AI features are shown in black and grey, respectively, and represented by the thickness of line. Exact proportions for each category are provided as numbers. Data for 2-4 hour time point for RNA are not included due to presence of maternal transcript at this stage. b. Top: Box plots show the distribution of effect sizes obtained from mixed linear models, for total counts. For each type of data (gene/feature), the effect sizes of time (T) and line (L) effects are shown. 
Bottom: Principal component analysis of gene expression for total counts. c. Top: Box plots showing the (gene/feature), the effect sizes of time (T) and line (L) effects are shown. Bottom: Principal component analysis of gene expression for allelic ratios. d. Results from mixed linear models examining the effect of developmental time versus line (genotype) between proximal and distal ATAC-seq peaks for total count data. Distal peaks show a larger time effect compared to genotype effect (Mann-Whitney U, $\mathrm{p}<2.2 \mathrm{e}-61$ ). This is only slightly evident for promoter proximal peaks (Mann-Whitney $U, p<8.5 e-5$ ).

Interactions between genetic and developmental effects can play an important role in gene 312 regulation (Paaby and Gibson 2016; Yadav et al. 2016). We therefore looked for evidence of

313 interaction effects in linear models fitted to total counts or allelic ratios containing only time,

314 only genotype, time plus genotype (time + genotype), or interactions between the two (genotype

$315 \mathrm{x}$ time $(\mathrm{GxT}))$. Interaction effects occur frequently at the total count level and are particularly

316 common for gene expression, making up nearly $30 \%$ of all analyzed models (Table S6) and

317 highlighting a potentially important role for developmental stage by genetic (GxT) interactions in

318 population-level variation during embryogenesis, as previously observed (Cannavo et al. 2017).

319 In contrast, there is little evidence for interaction effects at the level of allelic ratios for gene 320 expression or ATAC-seq peaks (Table S6), consistent with the relatively small numbers of allelic

321 ratios reported to show influences of gene $\mathrm{x}$ environment interactions across environmental

322 conditions (Moyerbrailean et al. 2016; Knowles et al. 2017).

323 In summary, allelic effects are often larger at distal compared to promoter regions, with allelic

324 effects at both regions being surprisingly stable across develompental time points. In contrast,

325 total counts vary dramatically between time points, with interactions between genotype and

326 developmental stage (GxT) being common, particularly for gene expression. Given that total

327 counts are influenced by genetic variation in both cis and trans, this highlights an important role

328 for trans acting variation in the maintenance of evolutionarily relevant interaction effects

\section{Information flows in different directions across cis-regulatory layers}

331 Although quantitative signals at chromatin features are highly correlated with gene

332 expression, the relative causal relationships between chromatin accessibility, histone

333 modifications, and gene expression remain unclear. To assess this, we used allelic ratios as a

334 perturbation measured at different regulatory layers to model the paths by which genetic 
335 variation influences regulatory phenotypes. Allelic ratios in all pairs of datatypes are correlated, 336 to varying extents (Fig. 4a), and in all cases we could reject the null hypothesis of independence

337 (e.g. highest p-value between all comparisons was 4.2e-17 for ATAC and H3K4me3). We tested

338 for an enrichment/depletion of co-occurring, statistically significantly imbalanced (FDR $<0.1)$

339 genes/features using an intersection-union test (Fig 4b; Methods), using a distance of $+/-1500 \mathrm{~kb}$

340 to assig distal features to genes. The co-occurrence of allelic imbalance is especially pronounced

341 for chromatin features, in particular H3K4me3 and proximal H3K27ac with a log-odds $>2.0$ (Fig.

342 4b). Interestingly, for chromatin accessibility and H3K27ac, the co-occurrence of AI is more

343 pronounced at promoters (proximal) than enhancers (distal) (Fig. 4b), despite allelic imbalance

344 being slightly more frequent ( $<<2.2 \mathrm{e}-16$, Fig. 2c) and of greater magnitude (Fig. S2b) at distal

345 sites. This suggests that H3K27ac and chromatin accessibility are more functionally coupled at

346 promoters compared to enhancers, perhaps reflecting the fact that not all active enhancers seem

347 to require H3K27ac (Bonn et al. 2012; Pradeepa et al. 2016).

348 Due to the large amount of covariation between the different regulatory features (Fig. $4 \mathrm{a}$ ), it is 349 difficult to infer causal relationships from correlation data alone. To address this, we used partial 350 correlation to identify independent, pairwise correlations between multiple co-varying variables

351 beyond their global correlations after thresholding on allelic ratios to remove features/genes with 352 low information content (Fig. 4c, S6a, Methods) (Lasserre et al. 2013; Pai et al. 2015). We first 353 analyzed the total count data to evaluate the overall relationships among histone modifications 354 and gene expression. Our results closely mirror those of Lasserre et al in CD4+ and IMR90 cells 355 (Lasserre et al. 2013), including finding a clear relationship between gene expression levels and 356 the total abundance of H3K27ac that 'explains away' (at least in a statistical sense) much of the 357 correlation between gene expression and promoter-proximal H3K4me3 (Fig. 4d, left). We also 358 observed a statistically significant relationship between open chromatin and gene expression, 359 though the strength of this partial correlation is reduced relative to standard Pearson correlation 360 analyses (Fig. S6b). 

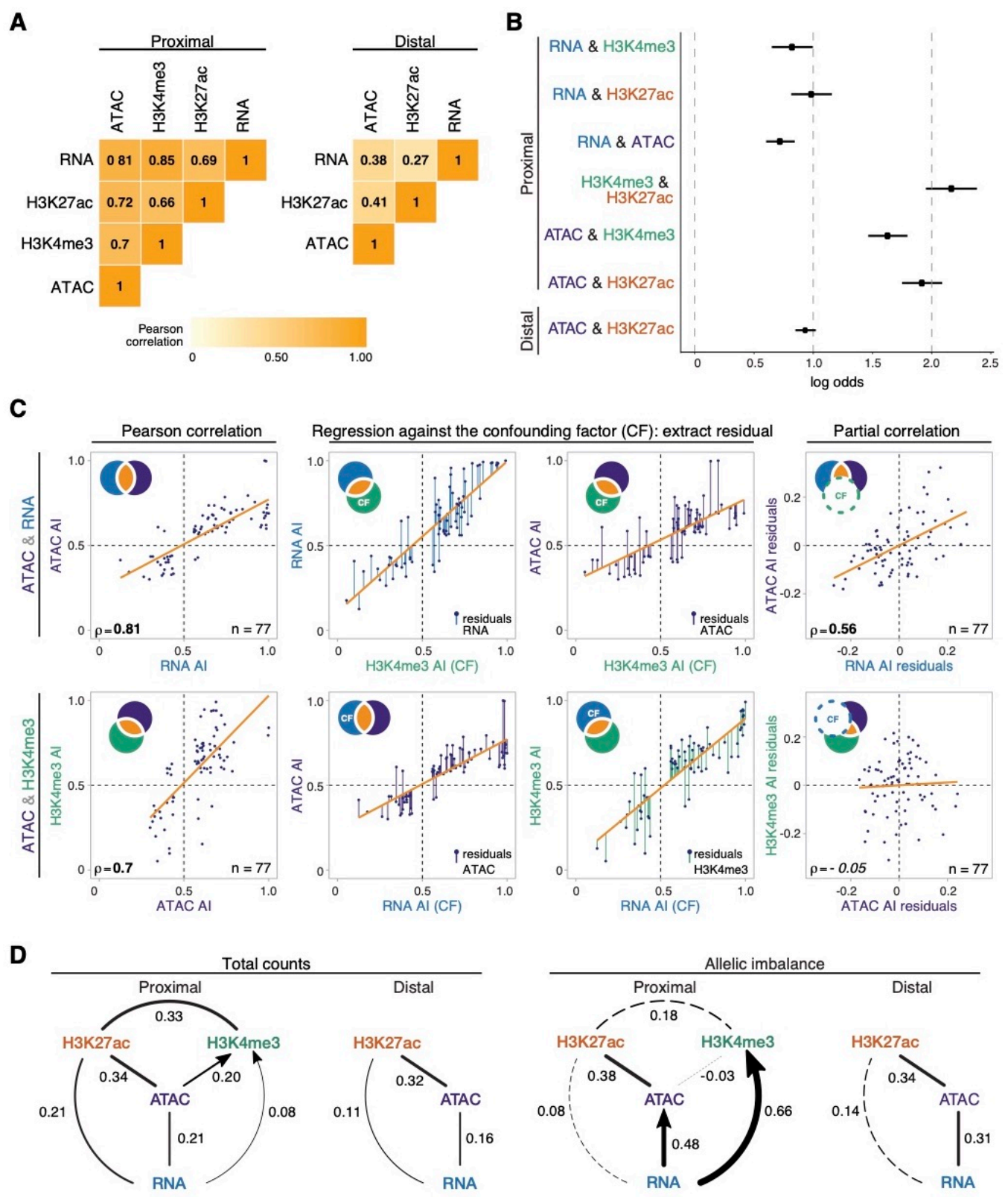

\section{Figure 4: Allelic imbalance is propagated through regulatory layers via different epigenetic paths}

a. Heatmaps show Pearson correlation coefficient of allelic ratios between each pair of data type for promoter proximal (left) and promoter distal (right) regions. Data restricted to 6-8 hours plus 10-12 hours, and features/genes whose allelic ratio exceeds $0.5+/-0.06$. b. Increased log odds of co-occurrence of allelic imbalance between two regulatory layers. X-axis shows the log odds based on intersection-union tests (Methods). 6-8 hours plus 10-12 hours data is shown. Bars stemming from dots are $95 \%$ confidence intervals. c. Stepwise example of a partial correlation analysis of allelic ratios for three variables (ATAC, RNA and $\mathrm{H} 3 \mathrm{~K} 4 \mathrm{me} 3$ ). Partial correlation analysis is shown between gene expression and chromatin accessibility (upper row), and promoter proximal H3K4me 3 and chromatin accessibility (lower row). Venn diagram schematics (top left) illustrates the variance of each variable and its shared proportion 
(orange), as measured by linear regression (orange lines). Left panels: Pearson correlations for the two comparisons are significant. Middle panels: regression of each initial variable against a third, confounding variable (H3K4me3, upper row; RNA, lower row). Residuals of the initial variables (colored lines) represent the non-overlapping part of the circle of the same color in the schematic. Right panels: correlations of the residuals, which exclude the variance shared by the confounding factor (dashed circle in schematic). This resulting partial correlation is not significant in the bottom example, suggesting a lack of direct correlation within the pair H3K4me3-ATAC. d. Partial correlation and directional dependency regression for total counts (left) and allelic ratios (right). Significant partial correlations (solid lines) suggest dependencies among regulatory layers. For each significant edge $(\mathrm{p}<0.01)$, copula regression was used to assign directionality (arrows, delta $>0.01$ ). Line thickness indicates the value of partial correlations, dashed lines indicate non-significance. Results for promoter proximal and distal regions shown separately.

To assess the functional impact of cis-regulatory perturbations, we next applied the partial correlations analysis to allelic ratios (Fig. 4d, right). Relative to the total count data, allelic ratios reveal a much stronger relationship between open chromatin and gene expression for both proximal and distal regulatory elements (Fig. 4d, right), highlighting an important, possibly causal, link between mutations affecting accessibility (presumably TF occupancy) and gene expression. A significant correlation is also observed between $\mathrm{H} 3 \mathrm{~K} 27 \mathrm{ac}$ and open chromatin at promoters, though interestingly, we see little evidence for a direct relationship between H3K27ac and gene expression itself (Fig. 4d, right). The latter is surprising as it differs from what is observed with total count data, and suggests that although promoter H3K27ac is highly correlated with, and even predictive of gene expression (Karlic et al. 2010), they may not be mechanistically directly linked. In contrast, allelic ratios for promoter proximal H3K4me3 show strong evidence of a direct correlation with gene expression that is independent of, at least in a statistical sense, allelic differences in chromatin accessibility or H3K27ac (Fig. 4d, right). Taken

374 together, this analysis suggests two independent pathways by which selection on segregating mutations could influence gene expression, one affecting open chromatin and promoter-proximal $\mathrm{H} 3 \mathrm{~K} 27 \mathrm{ac}$, and the other influencing H3K4me3.

377 To explore these relationships further, we analyzed each edge identified by partial correlations 378 using copula directional dependence analysis (Kim et al. 2008; Lee and Kim 2019), a statistical approach based on copula regression that evaluates the directionality of the pairwise relationships

380 allowing for non-linearities (Methods). This method assigns a direction to each edge for which

381 there is clear evidence for greater explanatory weight in one direction. For TSS-proximal 382 regions, this placed RNA upstream of both $\mathrm{H} 3 \mathrm{~K} 4 \mathrm{me} 3$ and open chromatin (Fig. 4d right, arrow). 
383 Although counter intuitive at first glance, this suggests that gene expression is not highly

384 sensitive to variations in $\mathrm{H} 3 \mathrm{~K} 4 \mathrm{me} 3$, while conversely changes to RNA is more predictive of

385 H3K4me3 enrichment. This could reflect redundancy between regulatory elements, i.e. changes

386 in a single open chromatin region, as tested here, may not be sufficient to impact expression in an

387 allele-specific manner. This result is also consistent with the hypothesis that H3K4me 3 is not

388 functionally required for transcription, but may rather be deposited as a consequence, and be

389 involved in post-transcriptional events, as recently proposed (Howe et al. 2017). Similarly,

390 allele-specific variation in RNA better explains variation in chromatin accessibility compared to

391 the reverse, i.e. not all variation in open chromatin leads to a corresponding change in gene

392 expression (Fig. 4d, right).

393 In summary, cis-acting genetic variation shows greater covariance between open chromatin

394 and H3K27ac enrichment at promoters compared to putative enhancers. By measuring

395 informative dependencies on the impact of cis-acting genetic variation, we identified multiple

396 epigenetic pathways affecting transcription. Specifically, genetic variation acts to change gene

397 expression levels via the interplay between at least two different promoter-proximal paths: open

398 chromatin and H3K27ac, or H3K4me3. Moreover, the flow of information suggests that gene

399 expression is often buffered against cis-acting mutations (presumably affecting TF binding) at

400 associated regulatory elements.

401

\section{Regulatory buffering varies depending on gene function and local chromatin architecture}

404 regulatory landscapes. Metabolic genes, for example, typically have relatively simple and more

405 compact regulatory landscapes with fewer enhancers that are generally located close to the

406 gene's promoter (Zabidi et al. 2015). TFs, in contrast, have many enhancers often with partially

407 overlapping spatial activity ("shadow enhancers") that are located at varying distances from the

408 gene's promoter (Spitz and Furlong 2012; Long et al. 2016). This additional regulatory

409 complexity is thought to make TFs more robust to deletions and sequence variation affecting

410 their regulatory elements (Xiong et al. 2002; Cretekos et al. 2008; Montavon et al. 2011;

411 Cannavo et al. 2016). To examine this, we assessed the extent to which allelic imbalance in the

412 expression of different gene categories is independent of, or decoupled from, imbalance in their 
413 associated regulatory elements, treating gene categories with greater independence as 'buffered'.

414 Among all comparisons of conditional probabilities, the expression of TFs, transmembrane

415 genes, ancient genes (conserved bilaterian processes), signalling pathway genes, secreted genes,

416 are most insensitive to imbalance in other regulatory layers (Fig. 5a). In contrast, genes and their

417 regulatory elements associated with cytoskeletal function, glycoproteins, and notably metabolism

418 show an increased sensitivity to allelic imbalance in other regulatory layers (Fig. 5a). Taken

419 together, our analyses suggest that in addition to purifying selection acting to remove genetic

420 variation, regulatory buffering helps to ensure robust expression of TFs and other developmental

421 regulatory factors from the effects of cis-acting mutations.

422 To more directly assess the relationship between buffering and regulatory complexity, we 423 compared the number of ATAC-seq peaks in a gene's regulatory domain $(+/-1.5 \mathrm{~kb}$ TSS) with

424 the probability of imbalance in that gene's expression. Imbalanced genes have fewer associated 425 ATAC-seq peaks genome-wide (Kruskal Wallis $\mathrm{p}=1.1 \mathrm{e}-16$, Fig 5b). This trend is particularly 426 striking for single-peak genes, which have significantly more AI than genes with multiple 427 associated regulatory elements (Mann-Whitney $U$ test, $p$-value=6.4e-6). Consistent with the 428 observation of transcriptional robustness (a lack of AI) for genes with multiple regulatory 429 elements, genes associated with partially redundant enhancers (or shadow enhancers) have a 430 modest reduction in the frequency of allelic imbalance compared to genes without (Fig. 5c, $431 x^{2}=5.3, \mathrm{p}=0.02$ ), based on a previously defined set of shadow enhancers for mesodermal genes 432 (Cannavo et al. 2016). We note, however, that this buffering is not absolute - even genes with 433 multiple regulatory elements are more likely to be imbalanced when multiple associated peaks 434 show unbalanced allelic ratios (Fig. 5d).

435 In summary, there is a relationship between a gene's regulatory complexity and the degree to 436 which its expression is influenced by non-coding genetic variation in its regulatory elements, 437 with more regulatory elements providing a degree of buffering against genetic perturbations. 438 Allelic imbalance at multiple enhancers in the vicinity of a gene can have a cumulative influence 439 on allele-specific gene expression. 

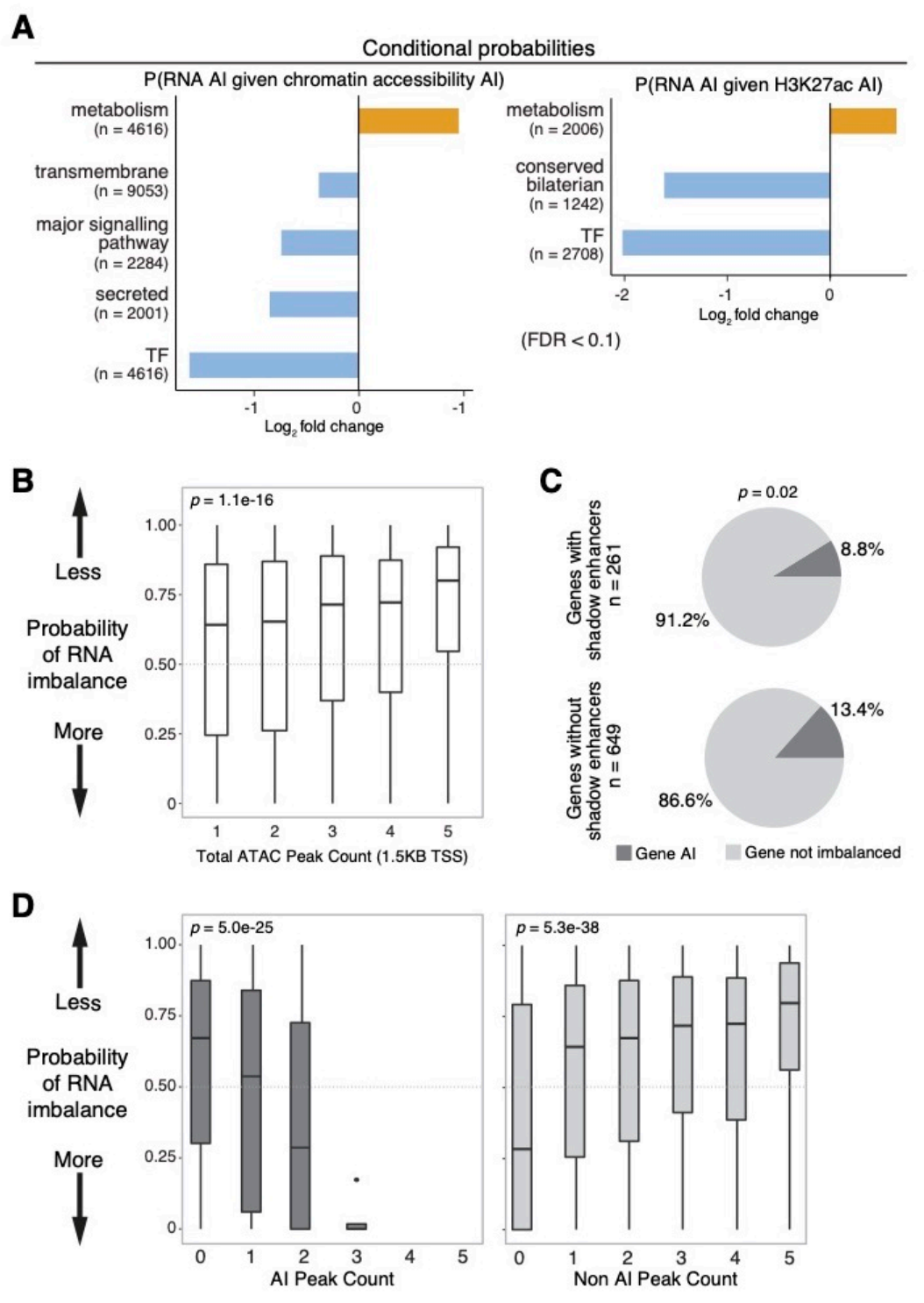

Figure 5: Regulatory buffering varies across gene categories and with local chromatin structure

a. Conditional probability of allelic imbalance in gene expression given allelic imbalance in associated chromatin peaks (left) and regions of H3K27ac (right) across gene categories. X-axis show $\log 2$ fold change where background is based on genome-wide expectation. Gene categories enriched (orange) or depleted (blue) for imbalance, relative to background, are indicated (FDR $>0.1$, Fisher's exact test). b. Box plots denote the probability of allelic imbalance in gene expression based on numbers of neighbouring ATAC peaks (TSS $<1.5 \mathrm{~kb}$ ). Genes associated to more ATAC peaks are more likely to show similar expression in both alleles compared to genes with fewer peaks. c. Pie charts displaying the proportion of genes with allelic imbalance in RNA associated to ATAC-seq peaks overlapping known partially redundant/shadow enhancers (top) or not (bottom). Genes associated with shadow enhancers are less likely to be allelic imbalanced compared to genes without $\left(x^{2}=5.3, \mathrm{p}=0.02\right)$. d. ATAC-seq peaks have a cumulative effect on gene expression. The probability of imbalance in gene expression (y-axis) is shown as a function of the number of ATAC-seq peaks that are allelic imbalanced (left) or not imbalanced (right). 


\section{Variation in gene expression is less heritable than for chromatin features}

441 Gene expression phenotypes are influenced by linked cis-acting genetic variation, but also by

442 trans acting variation that is not directly captured using F1s alone. To estimate the relative 443 impact of trans-acting variation, we performed the same experiments (iChIP-Seq, ATAC-Seq, 444 and RNA-Seq) on a trio of lines consisting of one F1 line and stage-matched embryos from the 445 maternal (vgn) and paternal (DGRP-399) lines. As the two homologous chromosomes in F1 446 cells have a common nuclear trans environment, allelic ratios in F1s estimate cis-based 447 differences between the two parents. Differences in parental read counts not reflected in F1 448 allelic-ratios give an estimate of trans-acting contributions to between line divergence (Landry et 449 al. 2005; Tirosh et al. 2009; Goncalves et al. 2012; Wong et al. 2017).

450 Using a maximum likelihood framework, we classified features as cis, trans, cistrans, or 451 conserved and found a similar distribution among categories for all non-coding chromatin 452 features, with cis-acting effects being more common than trans $(59 \%$ vs. $41 \%, \mathrm{p}<2.2 \mathrm{e}-16$, 453 chi^$^{\wedge}$; Fig. 6a, Methods, Table S7). This enrichment is particularly pronounced for histone 454 modifications, with nearly twice as many cis influenced peaks compared to trans (Fig 6a, S7a). 455 Gene expression, in contrast, is more strongly influenced by trans-acting genetic variation (Fig. 456 6a: $55 \%$ trans vs. $45 \%$ cis, $\mathrm{p}=0.0073$, chi $^{\wedge} 2$ ). Moreover, a higher fraction of cistrans genes 457 have more trans compared to cis variation, a pattern not observed for chromatin features (trans 458 proportions 0.67 vs. $0.53, \mathrm{p}=2.77 \mathrm{e}-05$; Fig. S7a).

459 Previous studies suggest that the effects of cis-acting mutations are more likely to be inherited 460 in an additive manner, compared to trans influences (Lemos et al. 2008; Meiklejohn et al. 2014; 461 Wong et al. 2017). This has important consequences, as it is typically additive genetic variation 462 that is most directly acted upon by natural selection (Lynch and Walsh 1998). We evaluated this

463 in our data by examining the extent to which the F1 signal (total read count) for each

464 gene/feature departed from the parental average (a strictly additive model). For open chromatin, 465 whether influenced by cis or trans, we could reject a non-additive model in fewer than $1 \%$ of 466 cases (Fig. S7b), consistent with the finding that most variation affecting TF binding is inherited 467 additively (Wong et al. 2017). For gene expression, in contrast, the additive model could be 468 rejected for $32 \%$ of genes, with trans influenced genes departing from an additive model far 469 more often than cis (Fig. 6b: 24\% vs. 2\%, p < 1e-4). 
A
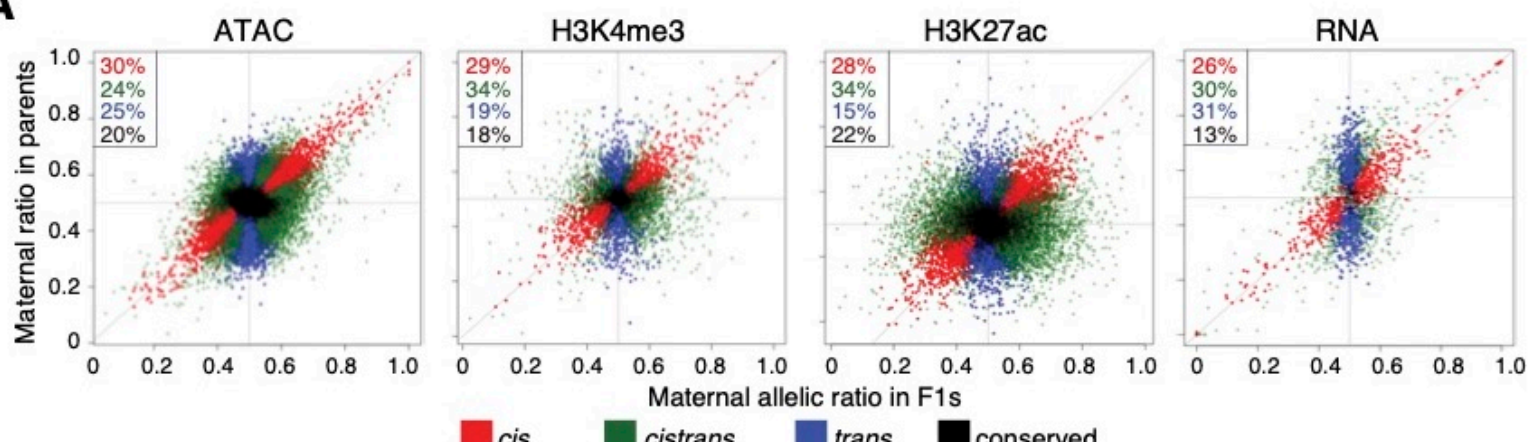

cis

cistrans

trans

B

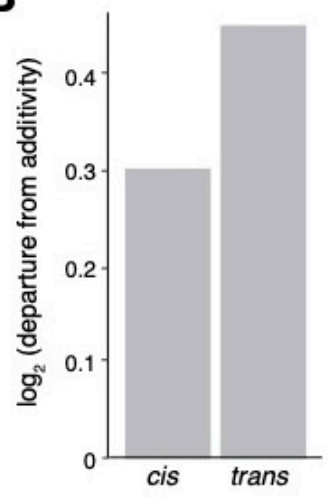

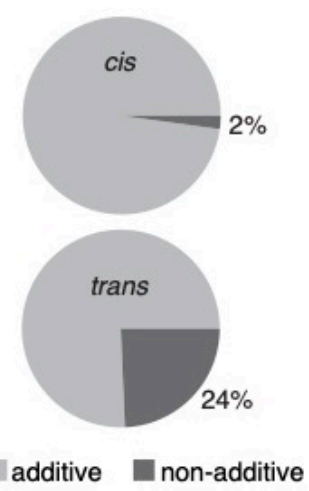

C

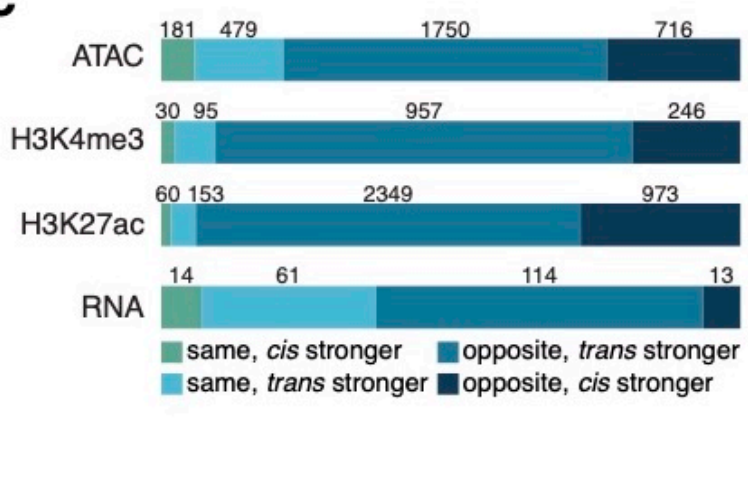

\section{Figure 6: Chromatin features are more heritable than gene expression}

a. Scatter plots of $\mathrm{F} 1$ allelic ratio (x-axis) against the maternal/paternal ratio observed in (normalized) parental, total count libraries. Genes/features along the diagonal are exclusively influenced by cis-acting variation, while vertically distributed genes/features show exclusively trans-influences. Colors indicate maximum likelihood classification into cis, trans, and cistrans (a mixture of cis and trans) or conserved genes/features. b. Left, bar plots shows the magnitude of deviation from additivity (parental mean) for features classified as cis vs. tran (BIC $>=2$ ). Right, pie charts showing fraction of additive and nonadditive genes for cis (upper) and trans (lower) classes. c. Classification of cistrans effects (BIC $>=2)$ for each regulatory layer into categories reflective of likely selective effects. Numbers and horizontal bars represent the size and relative proportions of each cistrans relative direction class in each data type. There is more directional selection (same directions, cis + trans $>$ cis) than compensatory evolution (opposite directions, cis + trans $<$ cis) in gene expression as compared to in chromatin features.

To better understand the factors that contribute to the proportion of trans-acting variation (and by association, non-additive heritability), we examined the contribution of regulatory complexity.

472 Mirroring our buffering results, genes with more regulatory elements in their vicinity are more

473 likely to be classified as trans-acting (trans $=2.58$ peaks per gene vs. $1.9, \mathrm{p}=0.00094)$ and more

474 likely to show non-additive inheritance (non-additively inherited genes $=2.19$ peaks per gene vs.

4751.82 peaks per gene, $p=1.4 \mathrm{e}-3$ ). Similarly, there is a significant, though modest, enrichment of 
476 trans influences among TFs and a depletion among metabolic genes, two categories that are

477 strongly distinguished in the complexity of their associated regulatory landscapes (Table S8).

478 Correspondingly, among 80 tested gene categories, DNA-binding TFs $(p=3 e-3)$ and

479 interestingly mitochondrial associated genes $(\mathrm{p}=2 \mathrm{e}-6)$ stand out as the two gene categories with

480 statistically elevated frequencies of non-additive inheritance (Fisher's exact test; Methods).

481 Thus, while TFs generally show reduced genetic variation among lines and reduced allelic

482 imbalance in gene expression (Fig. S3), they are still affected by trans-acting variants whose

483 non-additive inheritance reduces the efficacy by which selection can alter gene expression

484 differences among different lines.

485 Genes influenced by both cis and trans acting variants (cistrans) provide an opportunity to

486 understand patterns of recent selection: In the face of compensatory evolution, cis and trans

487 acting influences are more likely to work in opposite directions, while directional selection will

488 be more likely to reinforce cis and trans effects acting in the same direction. Using the

489 classification of cis and trans by Goncalves et al (Goncalves et al. 2012), we observed that cis

490 and trans effects were much more likely to act in a compensatory manner as compared to gene

491 expression: for chromatin accessibility and histone modifications, 13\% of cistrans features were

492 classified as same vs. 37\% for RNA (Fig. 6c: $\mathrm{p}<2.2 \mathrm{e}-16 \mathrm{chi}^{\wedge} 2$ ). This suggests that for RNA

493 there is either more frequent directional selection or less efficient selection against directional

494 changes. This result is robust to the method used to classify cis + trans effects (Landry et al.

495 2005), with $63 \%$ of cistrans RNA features being classified as divergent for RNA vs. $22 \%$ for

496 chromatin features (Fig, S7d: $\mathrm{p}<2.2 \mathrm{e}-16$ chi $^{\wedge} 2$ ). Taken together, these results suggest clear

497 differences in evolutionary trajectory between regulatory layers which reflects population

498 processes operating at different levels of organization, as well as differences between functional

499 gene classes.

500

501

502

503

504 


\section{Discussion}

506 We used genetic variation to better understand the impact of sequence variation in regulatory

507 DNA on embryonic gene expression, and to shed light on how these effects are propagated or

508 buffered through different layers of regulatory information. We generated allele-specific

509 measurements of chromatin occupancy (ATAC-seq), chromatin activity state (ChIP-seq of

510 chromatin modifications) and gene expression (RNA-seq) in F1 embryos from eight different

511 genotypes across multiple stages of embryogenesis. Our analysis of this extensive dataset led to

512 several conclusions about the impact of regulatory mutations on transcriptional phenotypes.

513 First, although cis-acting genetic variation in gene expression and associated regulatory

514 signals is fairly common in development, its effects are not equally distributed across the

515 genome. Allelic variation is both more frequent and has greater magnitude at distal regulatory

516 elements (putative enhancers) compared to promoters, despite genetic variation itself being more

517 common at promoters. This may in part be due to differences in the relative importance of

518 sequence content at promoters and enhancers - many promoters, particularly for broadly

519 expressed genes, are remarkably tolerant to mutations (Schor et al. 2017). Interestingly, despite

520 having a greater magnitude, AI at distal elements is less likely to be propagated to other

521 regulatory layers (Fig. 3), suggesting that enhancer mutations are either more effectively buffered

522 or of lower functional impact, a hypothesis that fits well with the observed robustness of gene

523 expression to deletions that remove distal regulatory elements (Hong et al. 2008; Cannavo et al.

524 2016). However, large effect gene-by-gene or gene-by-environment interactions can

525 theoretically serve to release this 'cryptic' genetic variation (Gibson and Dworkin 2004;

526 Schneider and Meyer 2017; Zheng et al. 2019). Whether such interactions are sufficiently

527 common for regulatory traits is currently unknown, although we note here that the genetic

528 contribution to (total count) gene expression varies considerably between time points, suggesting

529 a substantial context-specificity to the genetic variation underlying gene expression variation.

530 Second, although all data types (open chromatin, histone modifications, RNA levels) are

531 highly correlated, their explanatory values (potential causal relationships) as revealed by partial

532 correlation analysis are far from equal. Using cis-acting variation as perturbations to

533 development, we observed a strong, potentially direct relationship between genetic variants

534 affecting open chromatin (TF binding) at both proximal and distal sites and gene expression, as 
535 expected. The relationship between histone modifications and gene expression, however, proved 536 more surprising - in contrast to total count data, both in this study and previously reported

537 (Lasserre et al. 2013), we note a strong, potentially causal, link between allelic-imbalance in

538 H3K4me3 signal and allelic imbalance in associated genes.

539 Although highly correlated with gene expression, the functional requirement of $\mathrm{H} 3 \mathrm{~K} 4 \mathrm{me} 3$ for 540 transcription is controversial (Howe et al. 2017). Our copula analysis placed H3K4me3

541 downstream of RNA (Fig. 4d), suggesting that RNA levels are not impacted by variation

542 affecting $\mathrm{H} 3 \mathrm{~K} 4 \mathrm{me} 3$. This placement of RNA upstream of $\mathrm{H} 3 \mathrm{~K} 4 \mathrm{me} 3$, inferred from our

543 statistical analysis of the functional impact of genetic variation on both properties, is supported

544 by recent genetic ablation studies showing that RNA transcription does not require H3K4me3

545 (Clouaire et al. 2012; Margaritis et al. 2012; Clouaire et al. 2014). This is consistent with

546 suggestions that $\mathrm{H} 3 \mathrm{~K} 4 \mathrm{me} 3$ is deposited as a consequence of transcription and may be required in

547 more downstream post-transcriptional events (Howe et al. 2017). In addition, we also observed a

548 second, independent, pathway in which genetic mutations affecting H3K27ac impacted gene

549 expression, but only when they were also associated with cis-influenced changes in chromatin 550 accessibility.

551 Third, the impact of cis-regulatory variation on gene expression is influenced by regulatory 552 complexity, with genes that have more regulatory elements being less likely to show allelic 553 imbalance (Fig 5). In part, this may be due to selection against variation in regulatory elements 554 associated with these genes. As observed in other studies (Cannavo et al. 2016, ), we found a 555 clear reduction in allelic variation in regulatory elements associated with TFs and developmental 556 regulators as compared to other gene categories. But selection is unlikely to be the whole story. 557 Even when associated mutations are present, TFs and other genes with complex regulation show

558 a degree of independence from allelic imbalance in associated regulatory layers, an active 559 buffering process resulting from the presence of multiple regulatory inputs (Waymack 2019).

560 Notably, the buffering of genes with multiple regulatory elements is not absolute - as the number 561 of regulatory regions with AI near a gene increases, so does the probability that the gene shows 562 allelic imbalance. We propose that information averaging in cis-regulatory landscapes enhances 563 the overall consistency of transcriptional responses, with clustered regulatory elements, including 564 shadow enhancers, leading to a reduction in overall allelic imbalance. In addition, large effect 565 mutations can directly influence gene expression, with likely consequences for adaptive 
566 phenotypes, while small effect mutations, e.g. those affecting histone modifications or chromatin

567 accessibility without affecting gene expression, may accumulate over time to have functionally

568 relevant phenotypes.

569 Finally, we found that trans-acting variation is more common for RNA than for any other

570 regulatory layer, with resulting consequences for the selection and heritability of gene expression

571 relative to chromatin features. Specifically, genes with complex regulatory landscapes, e.g.

572 transcription factors, had a higher trans proportion of their overall genetic influences. This

573 observation, which is likely due to buffering effects within complex cis regulatory landscapes,

574 has potentially counter intuitive evolutionary consequences, as predominantly trans-influenced

575 genes are significantly more likely to show non-additive, and thus less selectable, patterns of

576 inheritance. As a result, trans-acting variation affecting genes such as TFs may remain in

577 populations even as negative selection and buffering act to reduce the influence of cis-acting

578 mutations.

579 In summary, allelic variation in chromatin accessibility and histone modifications at

580 regulatory elements is prevalent in the genome and capable of propagating across regulatory

581 layers. Information flow depends on the type of regulatory element and appears mitigated at

582 developmental factors. Notably, these cis-regulatory changes to individual genes do not have an

583 appreciable effect on overall developmental programs. 


\section{Methods}

587 Detailed methods for all sections are provided in the supplementary file.

\section{Fly husbandry, crosses and embryo collection}

590 F1 hybrid embryos were generated by crossing males from eight genetically distinct inbred lines

591 from the Drosophila Genetic Reference Panel (DGRP) collection (Mackay et al. 2012) to

592 females from a common maternal "virginizer" line. The virginizer line contains a heat-shock

593 inducible pro-apoptotic gene ( $h i d$ ) on the Y chromosome (Starz-Gaiano et al. 2001) of a

594 laboratory reference strain $\left(w^{1118}\right)$. We made the virginizer line isogenic by backcrossing for

595 over 20 generations (Ghavi-Helm et al. 2019). Placing embryos from the virginizer stock at $37^{\circ} \mathrm{C}$

596 kills all male embryos, thereby facilitating the collection of a large population of isogenic virgin

597 females, which were mated to males of different DGRP lines (listed in Fig. 1a). In addition, we

598 collected samples from the parents of one genotype (399) for cis-trans analysis (see below).

RNA-seq, ATAC-seq and iChIP

601 For three developmental stages (2-4hr, 6-8hr and 10-12hr after egg-laying), we performed RNA-

602 Seq, ATAC-Seq, and iChIP for H3K27ac and H3K4me3 for pooled embryos of each F1

603 strain. All experiments were performed in biological replicates from independent embryo

604 collections. iChIP experiments were performed as described in Lara-Astiaso et al. 2014 (Lara-

605 Astiaso et al. 2014). ATAC-seq libraries were 125bp PE, RNA-Seq 118bp PE, and iChIP 75bp

606 PE. In addition, gDNA from $\sim 100$ embryos per F1 cross was extracted and 75bp SE libraries

607 constructed. All libraries were run on a Bioanalyzer chip, multiplexed and sequenced with

608 Illumina machines.

609

610 Sequencing reads processing

611 Strain-specific genomes and liftOver chain files were constructed for each DGRP paternal line

612 using custom scripts to insert SNPs and indels into the Drosophila dm3 assembly (version 5 from

613 FlyBase). To annotate these parental genomes, we used pslMap (Zhu et al. 2007) to shift

614 reference annotations r5.57 to the parental genomes. ATAC-seq and ChIP-seq reads were

615 mapped using BWA (Li and Durbin 2010), while RNA-seq reads mapped using STAR (Dobin et 
616 al. 2013). In all cases, overlapping read pairs were trimmed so each base was covered only once

617 by the higher quality read. The resulting alignments against both parental genomes were merged

618 into a single alignment file. To generate allele-specific counts, reads were scored for their

619 overlap with known cross-specific SNPs. Discordant reads (those overlapping alleles assigned to

620 different parents) were discarded. Genomic DNA was generated for each F1 line to filter

621 potentially miscalled variants, and simulated reads from each parental genome were used to

622 assess and filter out regions with likely mappability errors. Peak calling was performed using

623 Macs2 (-broad) for iChIP reads and Hotspot for ATAC-seq reads (Zhang et al. 2008; John et al.

624 2011). To compare between lines and times, we constructed merged peak coordinates across

625 samples (Supplementary methods).

626

\section{Test for allele-specific imbalance}

628 Due to the extensive maternally deposited transcripts still present at 2-4 hours, we excluded the

629 RNA-seq data from this time point from all down-stream allele-specific analysis to avoid

630 potential confounding effects in allelic imbalance measurements. To test for allelic imbalance,

631 an empirical Bayesian method was used to test the null hypothesis for differences in read counts

632 between F1 alleles for each feature of each data set (RNA-seq, ATAC-seq, H3K4me3,

$633 \mathrm{H} 3 \mathrm{~K} 27 \mathrm{ac})$. Individual tests were performed for each line and for each time point. Total F1

634 counts $\left(n_{g}^{s, i, t}\right)$ can be modeled on an allele-specific basis $\left(z_{g}^{s, i, t}\right)$ using a beta-binomial

635 distribution. Specifically, $z_{g}^{s, i, t}$ denotes the number of reads from the maternal allele mapped to

636 feature f for pool of individuals $\mathrm{i}$, of paternal strain s, at time t. $n_{g}^{s, i, t}$ denotes the total number of

637 reads mapping to genes for pool of individuals $i$ of strain $s$, at time $t$.

$$
z_{f}^{s, i, t} \sim \operatorname{Bi}\left(n_{f}^{s, i, t}, p_{f}^{s, i, t}\right)
$$

$$
p_{g}^{s, i, t} \sim B e(\alpha, \beta)
$$

640 where $\alpha, \beta$ are the shape parameters of the beta distribution. We tested the following scenarios 641 by maximum likelihood estimation: 
644 Due to limited replicates per condition, we used information across features to reduce the

645 uncertainty of estimates and improve power by assuming that all features have the same mean-

646 variance relationship (Robinson et al. 2010; Love et al. 2014). Empirical data was used to

647 estimate the over-dispersion parameter $(\rho)$ for each data type based on the beta-binomial

648 distribution. Maximum likelihood estimation was used to obtain $\alpha$ and $\beta$ for each feature of time

$649 t$ and strain $s . \rho$ is calculated as follows:

$$
\rho=\frac{1}{\alpha+\beta+1}
$$

651 The mean over-dispersion value for all features was used as the shrinkage term and likelihood 652 ratio tests $(\mathrm{df}=1)$ were used to obtain a $\mathrm{p}$-value, which was adjusted using FDR (Benjamini

653 1995). Autosomes were tested separately to sex chromosomes; features on chromosome $\mathrm{X}$ were

654 tested using a background allelic ratio of no imbalance centered on the averaged ratio of maternal

655 versus paternal alleles across the data set being compared (i.e. RNA, ATAC, H3K4me3,

$656 \mathrm{H} 3 \mathrm{~K} 27 \mathrm{ac})$. Autosomal features were tested using a null distribution of 0.5 .

\section{Allele-specific changes across lines and developmental time}

659 A linear mixed-effects model, where random effect components were incorporated, was used to 660 estimate variability between pools of individuals, time points and lines,

$$
y_{f}^{d, s, r, t}=\mu_{f}+\delta_{f}^{t}+\omega_{f}^{s}+(\delta \omega)_{f}^{t} \quad \omega_{f}^{S} \sim \mathrm{N}\left(0, \sigma_{f}^{2}\right)
$$

$662 \mu_{f}$ is the intercept term. $\delta_{f}^{t}$ is a random effect term denoting time. $\omega_{f}^{s}$ is a random effect based on 663 strain and $(\delta \omega)_{f}^{t}$ is a interaction term for time by strain.

664 To infer the significance of time or strain dependent allele bias, we restricted the values that the 665 parameters can take. Library size differences were corrected for at the allele-combined count 666 level using the TMM method in 'edgeR' (Robinson et al. 2010) prior to analysis. Count data was 667 filtered for reads with more than 20 allele-combined counts. Each autosomal feature was tested 668 using read counts at SNPs common to all lines. Not all features contained enough information 669 for statistical testing, subsequent analyses were limited to features with at least six samples in 670 each of the three time points in at least four genetic strain. 


\section{Allele-specific changes across regulatory layers}

673 Intersection-union tests were used to examine the pairwise co-occurrence of allelic imbalance in

674 overlapping genes/features, limited to autosomes, based on rejecting the null hypothesis if a

675 significant outcome with respect to the feature compared at the same time point exists for both

676 data types (Berger 1996).

677 To infer pairwise relationships between regulatory data types while reducing indirect relations,

678 partial correlation analysis was performed using 'GeneNet' (Opgen-Rhein and Strimmer 2007)

679 for both allelic ratios and total count data. Directional dependence modeling was performed in a 680 regression framework using copulas to describe the bivariate distribution between our pairwise 681 datasets (Lee and Kim 2019). Copula regression was used to infer the flow of information for 682 pairwise relationships that showed a significant relationship in partial correlation analyses.

683 Conditional probabilities for the probability of allelic imbalance given imbalance in a different 684 regulatory data type were calculated by the definition:

$$
P(A \mid B)=\frac{P(A \cap B)}{P(B)}
$$

where $A$ and $B$ are the probabilities of allelic imbalance in each data type.

\section{Cis trans analysis}

689 For one F1 line (vgn x 399) and its parental lines, maximum likelihood estimation (MLE) was

690 used to compare parental and offspring ratios simultaneously to determine whether gene

691 expression, chromatin accessibility, H3K4me3 and H3K27ac enrichments are influenced by cis-,

692 trans-, conserved or both cis- and trans- acting effects by modeling read counts. For parents, the

693 data was modeled using negative binomial distributions and allelic differences in F1 alleles

694 modeled using beta-binomial distribution (Supplemental Methods). We constrained parameter

695 estimation for each model based on four different regulatory scenarios and derived maximum

696 likelihood values for each hypothetical case on a site-by-site basis. In the presentation of the

697 proportions of features assigned to each category (Fig. 6a, S7c), we presented the maximum

698 likelihood assignment. In subsequent analyses, we limited analyses to features that showed a

699 BIC difference $>=2$. 


\section{Test for compensatory mutation}

702 Genes were classified as having cis- and trans-acting influences following the procedure of

703 Goncalves et al. (Goncalves et al. 2012). For all genes, we asked if their cis and trans

704 contributes act to reinforce one another (same direction) or if they operated in opposite

705 directions. Formally, for the i_th gene, we define the average $\log 2$ fold change for the parental

706 lines as $\mathrm{x} \_\mathrm{i}$ and the average $\log 2$ allelic ratio from the $\mathrm{F} 1$ data as $\mathrm{y} \_\mathrm{i}$. We then classified:

707 Opposite - cis stronger: $\left(0<y_{-} i<x_{-} i\right)$ OR $\left(0>y_{-} i>x_{-} i\right)$

708 Opposite - trans stronger: $\left(\mathrm{x} \_\mathrm{i}_{-}<0<\mathrm{y} \_\mathrm{i}\right) \mathrm{OR}\left(\mathrm{y \_}<0<\mathrm{x} \_\mathrm{i}\right)$

709 Same - cis stronger $\left(0<x_{-} i<y \_i<2 x_{-} i\right)$ OR $\left(0>x_{-} i>y_{-} i>2 x_{-} i\right)$

710 Same - trans stronger $\left(0<2 x_{-} i<y \_i\right)$ OR $\left(0>2 x_{-} i>y \_i\right)$

711 A complementary analysis following Landry et al (Landry et al. 2005) can be found in the 712 supplemental methods.

\section{Measuring additive vs. non-additive heritability}

715 In the case of additively inherited gene expression (or read counts for any of our measured

716 features), the signal observed in the F1 is expected to be equal to the midpoint (average) of the

717 two parents, while non-additively inherited genes/features should show a significant departure

718 from that midpoint. To formally test for non-additivity, we made use of the standard workflow

719 in DESeq2 with two modifications. First, we set the betaPrior option equal to TRUE. After

720 setting the reference genotype to the F1 (vgn x 399) using the relevel function, we then extracted

721 the results using the 'results' function and the contrast vector $\mathrm{c}(0,1,-.5,-.5)$ to contrast the full

722 value of the F1 genotype with $1 / 2(\operatorname{vgn}+399)$. Features with an FDR $<.1$ were considered as

723 "non-additive". 


\section{Data Access}

727 All raw data has been deposited to EMBL-EBI hosted ArrayExpress, accession numbers:

728 E-MTAB-8877 (gDNA), E-MTAB-8878 (RNA-seq), E-MTAB-8879 (ATAC-seq), E-MTAB-

7298880 (ChIP-seq H3K4me3, H3K27ac). Processed data, including total counts, allelic ratios,

730 cis/trans estimates, estimated per-feature heritability, mappability filters, and parental genotype

731 files can all be downloaded from http://furlonglab.embl.de/data

\section{Acknowledgements}

734 We are very grateful to members of the Furlong lab for discussions and comments, in particular to

735 Olga Sigalova, Adam Rabinowitz, Marijn van Jaarsveld and Matteo Perino. This work was

736 technically supported by the EMBL Genomics Core Facility and the public resources of FlyBase,

737 BDGP, and RedFly. The work was financially supported by the European Research Council

738 (ERC advanced grant) agreement 322851 (CisRegVar) and 787611 (DeCRyPT) to E.E.F.

\section{Author Contributions}

741 EF, DG, and BZ conceived the project. BZ developed the ATAC-seq and RRV the iChIP protocol 742 for Drosophila embryos. BZ and RRV generated the data with help from DG. DG, SF, and EW 743 performed data analysis. DG and SF performed mapping bias analysis and allelic and total count 744 data processing. SF performed partial correlation analysis, and EW the statistical modeling for 745 allelic imbalance and Copula analysis. DG and EW performed cis/trans analysis. DG performed 746 analysis of heritability and evolutionary variation. EF, DG, EW, BZ and SF wrote the manuscript 747 with input from all authors. MT and DF helped to revise the manuscript.

\section{Disclosure Declaration}

750 The authors have no financial stake or conflicts of interest with the reported research. 


\section{References}

Ahituv N, Zhu Y, Visel A, Holt A, Afzal V, Pennacchio LA, Rubin EM. 2007. Deletion of ultraconserved elements yields viable mice. PLoS Biol 5: e234.

Battlay P, Schmidt JM, Fournier-Level A, Robin C. 2016. Genomic and Transcriptomic Associations Identify a New Insecticide Resistance Phenotype for the Selective Sweep at the Cyp6g1 Locus of Drosophila melanogaster. G3 (Bethesda) 6: 2573-2581.

Battle A, Khan Z, Wang SH, Mitrano A, Ford MJ, Pritchard JK, Gilad Y. 2015. Genomic variation. Impact of regulatory variation from RNA to protein. Science 347: 664-667.

Behera V, Evans P, Face CJ, Hamagami N, Sankaranarayanan L, Keller CA, Giardine B, Tan K, Hardison $\mathrm{RC}$, Shi J et al. 2018. Exploiting genetic variation to uncover rules of transcription factor binding and chromatin accessibility. Nat Commun 9: 782.

Benjamini Y, and Hochberg, Y. 1995. Controlling the False Discovery Rate: A Practical and Powerful Approach to Multiple Testing. Journal of the Royal Statistical Society: Series B (Methodological) 57: 289-300.

Berger RLaH, Jason C. 1996. Bioequivalence trials, intersection-union tests and equivalence confidence sets. Statistical Science 11: 283-302.

Bonn S, Zinzen RP, Girardot C, Gustafson EH, Perez-Gonzalez A, Delhomme N, Ghavi-Helm Y, Wilczynski B, Riddell A, Furlong EE. 2012. Tissue-specific analysis of chromatin state identifies temporal signatures of enhancer activity during embryonic development. Nat Genet 44: 148-156.

Borok MJ, Tran DA, Ho MC, Drewell RA. 2010. Dissecting the regulatory switches of development: lessons from enhancer evolution in Drosophila. Development 137: 5-13.

Brown CD, Johnson DS, Sidow A. 2007. Functional architecture and evolution of transcriptional elements that drive gene coexpression. Science 317: 1557-1560.

Buenrostro JD, Giresi PG, Zaba LC, Chang HY, Greenleaf WJ. 2013. Transposition of native chromatin for fast and sensitive epigenomic profiling of open chromatin, DNA-binding proteins and nucleosome position. Nat Methods 10: 1213-1218.

Bullaughey K. 2011. Changes in selective effects over time facilitate turnover of enhancer sequences. Genetics 187: 567-582.

Cannavo E, Khoueiry P, Garfield DA, Geeleher P, Zichner T, Gustafson EH, Ciglar L, Korbel JO, Furlong EE. 2016. Shadow Enhancers Are Pervasive Features of Developmental Regulatory Networks. Curr Biol 26: 38-51.

Cannavo E, Koelling N, Harnett D, Garfield D, Casale FP, Ciglar L, Gustafson HE, Viales RR, MarcoFerreres R, Degner JF et al. 2017. Genetic variants regulating expression levels and isoform diversity during embryogenesis. Nature 541: 402-406.

Celniker SE, Dillon LA, Gerstein MB, Gunsalus KC, Henikoff S, Karpen GH, Kellis M, Lai EC, Lieb JD, MacAlpine DM et al. 2009. Unlocking the secrets of the genome. Nature 459: 927-930.

Chen L, Ge B, Casale FP, Vasquez L, Kwan T, Garrido-Martin D, Watt S, Yan Y, Kundu K, Ecker S et al. 2016. Genetic Drivers of Epigenetic and Transcriptional Variation in Human Immune Cells. Cell 167: 1398-1414 e1324.

Clouaire T, Webb S, Bird A. 2014. Cfp1 is required for gene expression-dependent H3K4 trimethylation and H3K9 acetylation in embryonic stem cells. Genome Biol 15: 451.

Clouaire T, Webb S, Skene P, Illingworth R, Kerr A, Andrews R, Lee JH, Skalnik D, Bird A. 2012. Cfp1 integrates both $\mathrm{CpG}$ content and gene activity for accurate $\mathrm{H} 3 \mathrm{~K} 4 \mathrm{me} 3$ deposition in embryonic stem cells. Genes Dev 26: 1714-1728. 
Conrad T, Cavalli FM, Vaquerizas JM, Luscombe NM, Akhtar A. 2012. Drosophila dosage compensation involves enhanced Pol II recruitment to male X-linked promoters. Science 337: 742-746.

Core LJ, Waterfall JJ, Gilchrist DA, Fargo DC, Kwak H, Adelman K, Lis JT. 2012. Defining the status of RNA polymerase at promoters. Cell Rep 2: 1025-1035.

Cretekos CJ, Wang Y, Green ED, Martin JF, Rasweiler JJt, Behringer RR. 2008. Regulatory divergence modifies limb length between mammals. Genes Dev 22: 141-151.

Cusanovich DA, Reddington JP, Garfield DA, Daza RM, Aghamirzaie D, Marco-Ferreres R, Pliner HA, Christiansen L, Qiu X, Steemers FJ et al. 2018. The cis-regulatory dynamics of embryonic development at single-cell resolution. Nature 555: 538-542.

Daborn P, Boundy S, Yen J, Pittendrigh B, ffrench-Constant R. 2001. DDT resistance in Drosophila correlates with Cyp6g1 over-expression and confers cross-resistance to the neonicotinoid imidacloprid. Mol Genet Genomics 266: 556-563.

Dobin A, Davis CA, Schlesinger F, Drenkow J, Zaleski C, Jha S, Batut P, Chaisson M, Gingeras TR. 2013. STAR: ultrafast universal RNA-seq aligner. Bioinformatics 29: 15-21.

Doitsidou M, Flames N, Topalidou I, Abe N, Felton T, Remesal L, Popovitchenko T, Mann R, Chalfie M, Hobert O. 2013. A combinatorial regulatory signature controls terminal differentiation of the dopaminergic nervous system in C. elegans. Genes Dev 27: 1391-1405.

Epstein DJ. 2009. Cis-regulatory mutations in human disease. Brief Funct Genomic Proteomic 8: 310316.

Frankel N, Davis GK, Vargas D, Wang S, Payre F, Stern DL. 2010. Phenotypic robustness conferred by apparently redundant transcriptional enhancers. Nature 466: 490-493.

Garfield DA, Runcie DE, Babbitt CC, Haygood R, Nielsen WJ, Wray GA. 2013. The impact of gene expression variation on the robustness and evolvability of a developmental gene regulatory network. PLoS Biol 11: e1001696.

Georgiev P, Chlamydas S, Akhtar A. 2011. Drosophila dosage compensation: males are from Mars, females are from Venus. Fly (Austin) 5: 147-154.

Ghavi-Helm Y, Jankowski A, Meiers S, Viales RR, Korbel JO, Furlong EEM. 2019. Highly rearranged chromosomes reveal uncoupling between genome topology and gene expression. Nat Genet 51: $1272-1282$.

Gibson G, Dworkin I. 2004. Uncovering cryptic genetic variation. Nat Rev Genet 5: 681-690.

Goncalves A, Leigh-Brown S, Thybert D, Stefflova K, Turro E, Flicek P, Brazma A, Odom DT, Marioni JC. 2012. Extensive compensatory cis-trans regulation in the evolution of mouse gene expression. Genome Res 22: 2376-2384.

Hong JW, Hendrix DA, Levine MS. 2008. Shadow enhancers as a source of evolutionary novelty. Science 321: 1314.

Howe FS, Fischl H, Murray SC, Mellor J. 2017. Is H3K4me3 instructive for transcription activation? Bioessays 39: 1-12.

John S, Sabo PJ, Thurman RE, Sung MH, Biddie SC, Johnson TA, Hager GL, Stamatoyannopoulos JA. 2011. Chromatin accessibility pre-determines glucocorticoid receptor binding patterns. Nat Genet 43: 264-268.

Junion G, Spivakov M, Girardot C, Braun M, Gustafson EH, Birney E, Furlong EE. 2012. A transcription factor collective defines cardiac cell fate and reflects lineage history. Cell 148: 473-486.

Karlic R, Chung HR, Lasserre J, Vlahovicek K, Vingron M. 2010. Histone modification levels are predictive for gene expression. Proc Natl Acad Sci U S A 107: 2926-2931. 
Kasowski M, Grubert F, Heffelfinger C, Hariharan M, Asabere A, Waszak SM, Habegger L, Rozowsky J, 
884

885

886

887

888

889

890

891

892

893

894

895

896

897

898

899

900

901

902

903

904

905

906

907

908

909

910

911

912

913

914

915

916

917

918

919

920

921

922

923

924

925

926

927

Lu R, Rogan PK. 2018. Transcription factor binding site clusters identify target genes with similar tissuewide expression and buffer against mutations. F1000Res 7: 1933.

Lucchesi JC, Kuroda MI. 2015. Dosage compensation in Drosophila. Cold Spring Harb Perspect Biol 7: a019398.

Lynch M, Walsh B. 1998. Genetics and analysis of quantitative traits. Sinauer, Sunderland, Mass.

Mackay TF, Richards S, Stone EA, Barbadilla A, Ayroles JF, Zhu D, Casillas S, Han Y, Magwire MM, Cridland JM et al. 2012. The Drosophila melanogaster Genetic Reference Panel. Nature 482: 173178.

Margaritis T, Oreal V, Brabers N, Maestroni L, Vitaliano-Prunier A, Benschop JJ, van Hooff S, van Leenen D, Dargemont C, Geli V et al. 2012. Two distinct repressive mechanisms for histone 3 lysine 4 methylation through promoting 3'-end antisense transcription. PLoS Genet 8: e1002952.

Meiklejohn CD, Coolon JD, Hartl DL, Wittkopp PJ. 2014. The roles of cis- and trans-regulation in the evolution of regulatory incompatibilities and sexually dimorphic gene expression. Genome Res 24: 84-95.

Mi H, Vandergriff J, Campbell M, Narechania A, Majoros W, Lewis S, Thomas PD, Ashburner M. 2003. Assessment of genome-wide protein function classification for Drosophila melanogaster. Genome Res 13: 2118-2128.

Mikhaylichenko O, Bondarenko V, Harnett D, Schor IE, Males M, Viales RR, Furlong EEM. 2018. The degree of enhancer or promoter activity is reflected by the levels and directionality of eRNA transcription. Genes Dev 32: 42-57.

Montavon T, Soshnikova N, Mascrez B, Joye E, Thevenet L, Splinter E, de Laat W, Spitz F, Duboule D. 2011. A regulatory archipelago controls Hox genes transcription in digits. Cell 147: 1132-1145.

Moyerbrailean GA, Richards AL, Kurtz D, Kalita CA, Davis GO, Harvey CT, Alazizi A, Watza D, Sorokin Y, Hauff N et al. 2016. High-throughput allele-specific expression across 250 environmental conditions. Genome Res 26: 1627-1638.

Opgen-Rhein R, Strimmer K. 2007. From correlation to causation networks: a simple approximate learning algorithm and its application to high-dimensional plant gene expression data. BMC Syst Biol 1: 37.

Paaby AB, Gibson G. 2016. Cryptic Genetic Variation in Evolutionary Developmental Genetics. Biology (Basel) 5: E28.

Pai AA, Pritchard JK, Gilad Y. 2015. The genetic and mechanistic basis for variation in gene regulation. PLoS Genet 11: e1004857.

Pal K, Forcato M, Jost D, Sexton T, Vaillant C, Salviato E, Mazza EMC, Lugli E, Cavalli G, Ferrari F. 2019. Global chromatin conformation differences in the Drosophila dosage compensated chromosome X. Nat Commun 10: 5355.

Pradeepa MM, Grimes GR, Kumar Y, Olley G, Taylor GC, Schneider R, Bickmore WA. 2016. Histone H3 globular domain acetylation identifies a new class of enhancers. Nat Genet 48: 681-686.

Reddy TE, Gertz J, Pauli F, Kucera KS, Varley KE, Newberry KM, Marinov GK, Mortazavi A, Williams BA, Song L et al. 2012. Effects of sequence variation on differential allelic transcription factor occupancy and gene expression. Genome Res 22: 860-869.

Robinson MD, McCarthy DJ, Smyth GK. 2010. edgeR: a Bioconductor package for differential expression analysis of digital gene expression data. Bioinformatics 26: 139-140.

Schneider RF, Meyer A. 2017. How plasticity, genetic assimilation and cryptic genetic variation may contribute to adaptive radiations. Mol Ecol 26: 330-350. 
Schor IE, Degner JF, Harnett D, Cannavo E, Casale FP, Shim H, Garfield DA, Birney E, Stephens M, Stegle $\mathrm{O}$ et al. 2017. Promoter shape varies across populations and affects promoter evolution and expression noise. Nat Genet 49: 550-558.

Spitz F, Furlong EE. 2012. Transcription factors: from enhancer binding to developmental control. Nat Rev Genet 13: 613-626.

Spivakov M, Akhtar J, Kheradpour P, Beal K, Girardot C, Koscielny G, Herrero J, Kellis M, Furlong EE, Birney E. 2012. Analysis of variation at transcription factor binding sites in Drosophila and humans. Genome Biol 13: R49.

Starz-Gaiano M, Cho NK, Forbes A, Lehmann R. 2001. Spatially restricted activity of a Drosophila lipid phosphatase guides migrating germ cells. Development 128: 983-991.

Tirosh I, Reikhav S, Levy AA, Barkai N. 2009. A yeast hybrid provides insight into the evolution of gene expression regulation. Science 324: 659-662.

Turner LM, Chuong EB, Hoekstra HE. 2008. Comparative analysis of testis protein evolution in rodents. Genetics 179: 2075-2089.

Uhl JD, Zandvakili A, Gebelein B. 2016. A Hox Transcription Factor Collective Binds a Highly Conserved Distal-less cis-Regulatory Module to Generate Robust Transcriptional Outcomes. PLoS Genet 12: e1005981.

Urban J, Kuzu G, Bowman S, Scruggs B, Henriques T, Kingston R, Adelman K, Tolstorukov M, Larschan E. 2017. Enhanced chromatin accessibility of the dosage compensated Drosophila male $\mathrm{X}$-chromosome requires the CLAMP zinc finger protein. PLoS One 12: 0186855.

Villar D, Berthelot C, Aldridge S, Rayner TF, Lukk M, Pignatelli M, Park TJ, Deaville R, Erichsen JT, Jasinska AJ et al. 2015. Enhancer evolution across 20 mammalian species. Cell 160: 554-566.

Waszak SM, Delaneau O, Gschwind AR, Kilpinen H, Raghav SK, Witwicki RM, Orioli A, Wiederkehr M, Panousis NI, Yurovsky A et al. 2015. Population Variation and Genetic Control of Modular Chromatin Architecture in Humans. Cell 162: 1039-1050.

Waymack RF, A.; Enciso, G.; Wunderlich, Z. . 2019. Shadow enhancers suppress input transcription factor noise through distinct regulatory logic. bioRxiv doi:10.1101/778092.

Wittkopp PJ, Haerum BK, Clark AG. 2004. Evolutionary changes in cis and trans gene regulation. Nature 430: $85-88$.

Wittkopp PJ, Kalay G. 2011. Cis-regulatory elements: molecular mechanisms and evolutionary processes underlying divergence. Nat Rev Genet 13: 59-69.

Wong ES, Schmitt BM, Kazachenka A, Thybert D, Redmond A, Connor F, Rayner TF, Feig C, FergusonSmith AC, Marioni JC et al. 2017. Interplay of cis and trans mechanisms driving transcription factor binding and gene expression evolution. Nat Commun 8: 1092.

Xiong N, Kang C, Raulet DH. 2002. Redundant and unique roles of two enhancer elements in the TCRgamma locus in gene regulation and gammadelta T cell development. Immunity 16: 453-463.

Yadav A, Dhole K, Sinha H. 2016. Differential Regulation of Cryptic Genetic Variation Shapes the Genetic Interactome Underlying Complex Traits. Genome Biol Evol 8: 3559-3573.

Zabidi MA, Arnold CD, Schernhuber K, Pagani M, Rath M, Frank O, Stark A. 2015. Enhancer-corepromoter specificity separates developmental and housekeeping gene regulation. Nature 518: 556-559.

Zhang Y, Liu T, Meyer CA, Eeckhoute J, Johnson DS, Bernstein BE, Nusbaum C, Myers RM, Brown M, Li W et al. 2008. Model-based analysis of ChIP-Seq (MACS). Genome Biol 9: R137.

Zheng J, Payne JL, Wagner A. 2019. Cryptic genetic variation accelerates evolution by opening access to diverse adaptive peaks. Science 365: 347-353. 
973 Zheng W, Zhao H, Mancera E, Steinmetz LM, Snyder M. 2010. Genetic analysis of variation in 974 transcription factor binding in yeast. Nature 464: 1187-1191.

975 Zhu J, Sanborn JZ, Diekhans M, Lowe CB, Pringle TH, Haussler D. 2007. Comparative genomics search

976 for losses of long-established genes on the human lineage. PLoS Comput Biol 3: e247.

977 Zinzen RP, Girardot C, Gagneur J, Braun M, Furlong EE. 2009. Combinatorial binding predicts spatio978 temporal cis-regulatory activity. Nature 462: 65-70.

979 